\title{
Piecewise-regular maps
}

\author{
Wojciech Kucharz ${ }^{1}$
}

Received: 27 April 2017 / Revised: 24 September 2017 / Published online: 20 October 2017 (C) The Author(s) 2017. This article is an open access publication

\begin{abstract}
Let $V, W$ be real algebraic varieties (that is, up to isomorphism, real algebraic sets), and $X \subseteq V$ some subset. A map from $X$ into $W$ is said to be regular if it can be extended to a regular map defined on some Zariski locally closed subvariety of $V$ that contains $X$. Furthermore, a continuous map $f: X \rightarrow W$ is said to be piecewise-regular if there exists a stratification $\mathscr{S}$ of $V$ such that for every stratum $S \in \mathscr{S}$ the restriction of $f$ to each connected component of $X \cap S$ is a regular map. By a stratification of $V$ we mean a finite collection of pairwise disjoint Zariski locally closed subvarieties whose union is equal to $V$. Assuming that the subset $X$ of $V$ is compact, we prove that every continuous map from $X$ into a Grassmann variety or a unit sphere can be approximated by piecewise-regular maps. As an application, we obtain a variant of the algebraization theorem for topological vector bundles. If the variety $V$ is compact and nonsingular, we prove that each continuous map from $V$ into a unit sphere is homotopic to a piecewise-regular map of class $\mathscr{C}^{k}$, where $k$ is an arbitrary nonnegative integer.
\end{abstract}

Mathematics Subject Classification 14P05 $\cdot 14 \mathrm{P} 99 \cdot 57 \mathrm{R} 22$

Communicated by Ngaiming Mok.

$凶$ Wojciech Kucharz

Wojciech.Kucharz@im.uj.edu.pl

1 Faculty of Mathematics and Computer Science, Institute of Mathematics, Jagiellonian University, Łojasiewicza 6, 30-348 Kraków, Poland 


\section{Introduction}

In this paper, by a real algebraic variety we mean a locally ringed space isomorphic to an algebraic subset of $\mathbb{R}^{m}$, for some $m$, endowed with the Zariski topology and the sheaf of real-valued regular functions (such an object is called an affine real algebraic variety in [6]). The class of real algebraic varieties is identical with the class of quasi-projective real algebraic varieties, cf. [6, Proposition 3.2.10, Theorem 3.4.4]. Morphisms of real algebraic varieties are called regular maps. Each real algebraic variety carries also the Euclidean topology, induced by the standard metric on $\mathbb{R}$. Unless explicitly stated otherwise, we always assume that real algebraic varieties and their subsets are endowed with the Euclidean topology.

Topological properties of regular maps and their applications to algebraization of topological vector bundles were investigated in numerous papers [2-14, 16-25, 27,32$35,37,38,40-43,47-49,51,53,56,64,65,68,71,72,76-80,82,83]$. In general, regular maps are too rigid to reflect adequately topological phenomena. It is therefore desirable to introduce maps which have many good features of regular maps but are more flexible.

We first generalize the definition of regular map.

Definition 1.1 Let $V, W$ be real algebraic varieties, $X \subseteq V$ some (nonempty) subset, and $Z$ the Zariski closure of $X$ in $V$.

A map $f: X \rightarrow W$ is said to be regular if there exist a Zariski open neighborhood $Z_{0} \subseteq Z$ of $X$ and a regular map $\tilde{f}: Z_{0} \rightarrow W$ such that $\left.\tilde{f}\right|_{X}=f$.

The next step requires the concept of stratification. By a stratification of a real algebraic variety $V$ we mean a finite collection of pairwise disjoint Zariski locally closed subvarieties (some possibly empty) whose union is equal to $V$.

Definition 1.2 Let $V, W$ be real algebraic varieties, $f: X \rightarrow W$ a continuous map defined on some subset $X \subseteq V$, and $\mathscr{S}$ a stratification of $V$.

The map $f$ is said to be $\mathscr{S}$-regular if for every stratum $S \in \mathscr{S}$ the restriction of $f$ to $X \cap S$ is a regular map. Also, $f$ is said to be piecewise $\mathscr{S}$-regular if for every stratum $S \in \mathscr{S}$ the restriction of $f$ to each connected component of $X \cap S$ is a regular map (when $X \cap S \neq \varnothing$ ).

Moreover, $f$ is said to be stratified-regular (resp. piecewise-regular) if it is $\mathscr{T}$ regular (resp. piecewise $\mathscr{T}$-regular) for some stratification $\mathscr{T}$ of $V$.

Essentially, these notions do not depend on the ambient variety $V$. More precisely, suppose that $V$ is a Zariski locally closed subvariety of a real algebraic variety $V^{\prime}$. The map $f: X \rightarrow W$ is $\mathscr{S}$-regular (resp. piecewise $\mathscr{S}$-regular) if and only if it is $\mathscr{S}^{\prime}$-regular (resp. piecewise $\mathscr{S}^{\prime}$-regular), where $\mathscr{S}^{\prime}$ is the stratification of $V^{\prime}$ defined by $\mathscr{S}^{\prime}=\mathscr{S} \cup\{\bar{V} \backslash V\} \cup\left\{V^{\prime} \backslash \bar{V}\right\}$ with $\bar{V}$ the Zariski closure of $V$ in $V^{\prime}$. Conversely, given a stratification $\mathscr{P}$ of $V^{\prime}$, the map $f$ is $\mathscr{P}$-regular (resp. piecewise $\mathscr{P}$-regular) if and only if it is $\mathscr{P}(V)$-regular (resp. piecewise $\mathscr{P}(V)$-regular), where $\mathscr{P}(V)$ is the stratification of $V$ defined by $\mathscr{P}(V)=\{V \cap P: P \in \mathscr{P}\}$. Thus, in the definition of stratified-regular map (resp. piecewise-regular map) it does not matter whether $X$ is regarded as a subset of $V$ or as a subset of $V^{\prime}$.

Evidently, each stratified-regular map is piecewise-regular, whereas the converse is not always true. General properties of these two classes of maps and relationships 
between them are discussed in Sect. 2. Stratified-regular maps and functions are thoroughly investigated, in a more restrictive framework, in $[4,29,30,44,45,50,52,54$, $55,58,60-62,66,74,75,84]$, where they sometimes appear under different names (cf. Remark 2.5). If $X$ is a semialgebraic set, then each piecewise-regular map defined on $X$ is semialgebraic. Theorem 2.9 provides a nontrivial characterization of piecewiseregular maps among semialgebraic ones.

In this section we concentrate on topological properties of piecewise-regular maps. With notation as in Definition 1.2, we let $\mathscr{C}(X, W)$ denote the space of all continuous maps from $X$ into $W$, endowed with the compact-open topology. We say that the map $f$ can be approximated by piecewise $\mathscr{S}$-regular maps if every neighborhood of $f$ in $\mathscr{C}(X, W)$ contains a piecewise $\mathscr{S}$-regular map. Approximation of $f$ by maps of other types (regular, $\mathscr{S}$-regular, stratified-regular, piecewise-regular, etc.) is defined in the analogous way.

We pay special attention to maps with values in Grassmannians. We let $\mathbb{F}$ stand for $\mathbb{R}, \mathbb{C}$ or $\mathbb{H}$ (the quaternions). When convenient, $\mathbb{F}$ will be identified with $\mathbb{R}^{d(\mathbb{F})}$, where $d(\mathbb{F})=\operatorname{dim}_{\mathbb{R}} \mathbb{F}$. We will consider only left $\mathbb{F}$-vector spaces, which plays a role if $\mathbb{F}=\mathbb{H}$ since the quaternions are noncommutative. For any integers $r$ and $n$, with $0 \leq r \leq n$, we denote by $\mathbb{G}_{r}\left(\mathbb{F}^{n}\right)$ the Grassmann space of $r$-dimensional $\mathbb{F}$-vector subspaces of $\mathbb{F}^{n}$. As in [6, Sections 3.4 and 13.3], $\mathbb{G}_{r}\left(\mathbb{F}^{n}\right)$ will be regarded as a real algebraic variety. The disjoint union

$$
\mathbb{G}\left(\mathbb{F}^{n}\right)=\coprod_{r=0}^{n} \mathbb{G}_{r}\left(\mathbb{F}^{n}\right)
$$

is also a real algebraic variety.

Theorem 1.3 Let $V$ be a real algebraic variety and let $X \subseteq V$ be a compact subset. Then, for each positive integer $n$, every continuous map from $X$ into $\mathbb{G}\left(\mathbb{F}^{n}\right)$ can be approximated by piecewise-regular maps.

Under an additional assumption on $X$, we also have a stronger result.

Theorem 1.4 Let $V$ be a real algebraic variety and let $X \subseteq V$ be a compact locally contractible subset. Then there exists a stratification $\mathscr{S}$ of $V$ such that, for each positive integer $n$, every continuous map from $X$ into $\mathbb{G}\left(\mathbb{F}^{n}\right)$ can be approximated by piecewise $\mathscr{S}$-regular maps.

Virtually all topological spaces one encounters in real algebraic geometry are locally contractible; for example, any semialgebraic set is locally contractible, cf. [6, Theorem 9.3.6].

The proofs of Theorems 1.3 and 1.4, given in Sect. 4, are based on some fairly explicit constructions.

It is well-known that maps with values in $\mathbb{G}\left(\mathbb{F}^{n}\right)$ encode information on algebraic and topological $\mathbb{F}$-vector bundles, cf. [6,39]. This is also the case for stratified-algebraic $\mathbb{F}$-vector bundles introduced in [61] and further investigated in $[57,59,63,66]$. Theorems 1.3 and 1.4 have a bearing on $\mathbb{F}$-vector bundles as well, which is elaborated upon in Sect. 5. The main results of Sect. 5 are Theorems 5.10 and 5.11. 
We also have an approximation theorem for maps with values in the unit $n$-sphere

$$
\mathbb{S}^{n}=\left\{\left(u_{0}, \ldots, u_{n}\right) \in \mathbb{R}^{n+1}: u_{0}^{2}+\cdots+u_{n}^{2}=1\right\} .
$$

Theorem 1.5 Let $V$ be a real algebraic variety and let $X \subseteq V$ be a compact subset. Then, for each positive integer $n$, every continuous map from $X$ into $\mathbb{S}^{n}$ can be approximated by piecewise-regular maps.

Theorem 1.5, which is proved in Sect. 6, implies that each continuous map from $X$ into $\mathbb{S}^{n}$ is homotopic to a piecewise-regular map. However, the following homotopy result requires a different proof.

Theorem 1.6 Let $V$ be a compact nonsingular real algebraic variety, $n$ a positive integer, and $f: V \rightarrow \mathbb{S}^{n}$ a continuous map. Then there exists a stratification $\mathscr{S}$ of $V$ such that, for each nonnegative integer $k$, the map $f$ is homotopic to a piecewise $\mathscr{S}$-regular map $g: V \rightarrow \mathbb{S}^{n}$ of class $\mathscr{C}^{k}$.

We prove Theorem 1.6 in Sect. 7. It turns out that a suitable stratification $\mathscr{S}$ of $V$ is quite simple and consists of at most 3 strata.

Theorems 1.3, 1.5 and 1.6 are optimal in the sense explained in the following example.

Example 1.7 Let $W$ be a compact nonsingular real algebraic variety. A cohomology class in $H^{q}(W ; \mathbb{Z} / 2)$ is said to be algebraic if the homology class Poincaré dual to it can be represented by a Zariski closed subvariety of $W$ of codimension $q$, cf. [3,6]. The set $H_{\mathrm{alg}}^{q}(W ; \mathbb{Z} / 2)$ of all algebraic cohomology classes in $H^{q}(W ; \mathbb{Z} / 2)$ forms a subgroup. Obviously, the unique generator $s_{q}$ of $H^{q}\left(\mathbb{S}^{q} ; \mathbb{Z} / 2\right) \cong \mathbb{Z} / 2$ is an algebraic cohomology class.

The real algebraic varieties $\mathbb{G}_{1}\left(\mathbb{F}^{2}\right)$ and $\mathbb{S}^{d(\mathbb{F})}$ are canonically biregularly isomorphic and will be identified. For any positive integer $m$, let $\mathbb{T}^{m}=\mathbb{S}^{1} \times \cdots \times \mathbb{S}^{1}$ be the $m$-fold product of $\mathbb{S}^{1}$. Clearly,

$$
\mathbb{T}^{m} \subseteq \mathbb{R}^{2 m}=\mathbb{R}^{2 m} \times\{0\} \subseteq \mathbb{R}^{2 m+1}
$$

We will regard $\mathbb{T}^{m}$ as a subset of $\mathbb{R}^{2 m+1}$.

Fix an integer $m \geq d(\mathbb{F})+1$, and let $y_{0}$ be a point in $\mathbb{T}^{m-d(\mathbb{F})}$. Let $\alpha$ be the homology class in $H_{d(\mathbb{F})}\left(\mathbb{T}^{m} ; \mathbb{Z} / 2\right)$ represented by the $\mathscr{C}^{\infty}$ submanifold $N:=\mathbb{T}^{d(\mathbb{F})} \times\left\{y_{0}\right\} \subseteq \mathbb{T}^{m}$. Set

$$
G:=\left\{u \in H^{d(\mathbb{F})}\left(\mathbb{T}^{m} ; \mathbb{Z} / 2\right):\langle u, \alpha\rangle=0\right\},
$$

where $\langle u, \alpha\rangle$ stands for the Kronecker product. Let $\pi: \mathbb{T}^{m}=\mathbb{T}^{d(\mathbb{F})} \times \mathbb{T}^{m-d(\mathbb{F})} \rightarrow$ $\mathbb{T}^{d(\mathbb{F})}$ be the canonical projection and let $\tau: \mathbb{T}^{d(\mathbb{F})} \rightarrow \mathbb{S}^{d(\mathbb{F})}$ be a $\mathscr{C}^{\infty}$ map of topological degree 1 . For the $\mathscr{C}^{\infty}$ map $g:=\tau \circ \pi: \mathbb{T}^{m} \rightarrow \mathbb{S}^{d(\mathbb{F})}$, we have

$$
g^{*}\left(s_{d(\mathbb{F})}\right) \notin G .
$$

Since the normal bundle to $N$ in $\mathbb{T}^{m}$ is trivial and $N$ is the boundary of a compact $\mathscr{C}^{\infty}$ manifold with boundary, it follows from [23, Proposition 2.5, Theorem 2.6] that there 
exist a nonsingular real algebraic variety $V$ and a $\mathscr{C}^{\infty}$ diffeomorphism $\sigma: V \rightarrow \mathbb{T}^{m}$ with

$$
H_{\mathrm{alg}}^{d(\mathbb{F})}(V ; \mathbb{Z} / 2) \subseteq \sigma^{*}(G) .
$$

The map

$$
h:=g \circ \sigma: V \rightarrow \mathbb{G}_{1}\left(\mathbb{F}^{2}\right)=\mathbb{S}^{d(\mathbb{F})}
$$

is of class $\mathscr{C}^{\infty}$, and

$$
h^{*}\left(s_{d(\mathbb{F})}\right) \notin H_{\mathrm{alg}}^{d(\mathbb{F})}(V ; \mathbb{Z} / 2) .
$$

By [61, Propositions 7.2 and 7.7], $h$ is not homotopic to any stratified-regular map. In particular, $h$ cannot be approximated by stratified-regular maps, which is of interest in view of Theorems 1.3 and 1.5. Also, by Proposition 2.13, $h$ is not homotopic to any piecewise-regular map of class $\mathscr{C}^{\infty}$; thus the case $k=\infty$ cannot be included in Theorem 1.6.

Furthermore, according to [15, Proposition 1.2], $V$ as above can be assumed to be a Zariski closed subvariety of $\mathbb{R}^{2 m+1}$ that is obtained from $\mathbb{T}^{m}$ via an arbitrarily small $\mathscr{C}^{\infty}$ isotopy.

From a different viewpoint, strengthening of Theorems 1.3, 1.5 and 1.6 might be possible. Given a compact nonsingular real algebraic variety $V$ and two positive integers $n$ and $k$, it remains an open question whether every continuous map from $V$ into $\mathbb{G}\left(\mathbb{F}^{n}\right)$ or $\mathbb{S}^{n}$ can be approximated by piecewise-regular maps of class $\mathscr{C}^{k}$.

Example 1.8 Stratified-regular maps often have better approximation and homotopy properties than regular ones. For instance, if $V$ is a compact nonsingular real algebraic variety of dimension $n \geq 1$, then every continuous map from $V$ into $\mathbb{S}^{n}$ can be approximated by stratified-regular maps, cf. [55, Corollary 1.3]. On the other hand, if $n$ is even, then each regular map from $\mathbb{T}^{n}$ into $\mathbb{S}^{n}$ is null homotopic, cf. [8] or [6, Theorem 13.5.1].

Piecewise-regular maps are not always more flexible than regular maps.

Example 1.9 Let $F_{r}$ be the Fermat curve of degree $r$ in the real projective plane $\mathbb{P}^{2}(\mathbb{R})$,

$$
F_{r}:=\left\{(x: y: z) \in \mathbb{P}^{2}(\mathbb{R}): x^{r}+y^{r}=z^{r}\right\} .
$$

Clearly, $F_{2}$ can be identified with $\mathbb{S}^{1}$. If $s>r \geq 2$, then every piecewise-regular map from $F_{r}$ into $F_{s}$ is constant. This claim holds since, by virtue of the Hurwitz-Riemann theorem [31, p. 140], every rational map from $F_{r}$ into $F_{s}$ is constant.

It would be of interest to decide whether or not counterparts of Theorems 1.3 and 1.5 hold for maps with values in an arbitrary rational nonsingular real algebraic variety.

We have already indicated how the present paper is organized. It should be added that Sect. 3 contains some preliminary technical results.

Henceforth, the following notation will be frequently used.

Notation 1.10 For any function $f: \Omega \rightarrow \mathbb{R}$ defined on some set $\Omega$, we put

$$
Z(f):=\{x \in \Omega: f(x)=0\} .
$$




\section{General properties of piecewise-regular maps}

We first deal with regular maps in the sense of Definition 1.1.

Lemma 2.1 Let $V$ be a real algebraic variety, $W \subseteq \mathbb{R}^{p}$ a Zariski closed subvariety, and

$$
f=\left(f_{1}, \ldots, f_{p}\right): X \rightarrow W \subseteq \mathbb{R}^{p}
$$

a map defined on some subset $X \subseteq V$. Then the following conditions are equivalent:

(a) The map $f$ is regular.

(b) Each component function $f_{j}: X \rightarrow \mathbb{R}$ is regular, $j=1, \ldots, p$.

Proof It is clear that (a) implies (b).

Suppose that (b) holds, and let $Z$ be the Zariski closure of $X$ in $V$. We can find a Zariski open neighborhood $Z_{0} \subseteq Z$ of $X$ and regular functions $\tilde{f}_{j}: Z_{0} \rightarrow \mathbb{R}$ such that $\left.\tilde{f}_{j}\right|_{X}=f_{j}$ for $j=1, \ldots, p$. The regular map $\tilde{f}=\left(\tilde{f}_{1}, \ldots, \tilde{f}_{p}\right): Z_{0} \rightarrow \mathbb{R}^{p}$ is continuous in the Zariski topology and $\tilde{f}(X) \subseteq W$. Hence $\tilde{f}\left(Z_{0}\right) \subseteq W$, which implies (a).

Regular functions can be characterized as follows.

Lemma 2.2 Let $V \subseteq \mathbb{R}^{n}$ be a Zariski closed subvariety, and $f: X \rightarrow \mathbb{R}$ a function defined on some subset $X \subseteq V$. Then the following conditions are equivalent:

(a) The function $f$ is regular.

(b) For each point $x \in X$ there exist a Zariski open neighborhood $V_{x} \subseteq V$ of $x$ and a regular function $F_{x}: V_{x} \rightarrow \mathbb{R}$ such that $F_{x}=f$ on $X \cap V_{x}$.

(c) There exist two regular functions $\varphi, \psi: V \rightarrow \mathbb{R}$ such that $X \subseteq V \backslash Z(\psi)$ and $f=\varphi / \psi$ on $X$.

(d) For each point $x \in X$ there exist a Zariski open neighborhood $U_{x} \subseteq \mathbb{R}^{n}$ of $x$ and two polynomial functions $P_{x}, Q_{x}: \mathbb{R}^{n} \rightarrow \mathbb{R}$ such that $U_{x} \subseteq \mathbb{R}^{n} \backslash Z\left(Q_{x}\right)$ and $f=P_{x} / Q_{x}$ on $X \cap U_{x}$.

(e) There exist two polynomial functions $P, Q: \mathbb{R}^{n} \rightarrow \mathbb{R}$ such that $X \subseteq \mathbb{R}^{n} \backslash Z(Q)$ and $f=P / Q$ on $X$.

Proof It readily follows that

$$
\text { (a) } \Rightarrow \text { (b) } \Rightarrow \text { (d), (e) } \Rightarrow \text { (c) } \Rightarrow \text { (a), and (e) } \Rightarrow \text { (d). }
$$

Suppose that (d) holds. For each point $x \in X$, pick a polynomial function $S_{x}: \mathbb{R}^{n} \rightarrow \mathbb{R}$ with $Z\left(S_{x}\right)=\mathbb{R}^{n} \backslash U_{x}$. Since the Zariski topology on $\mathbb{R}^{n}$ is Noetherian, we can find a finite subset $\left\{x_{1}, \ldots, x_{r}\right\} \subseteq X$ such that

$$
X \subseteq U:=U_{x_{1}} \cup \cdots \cup U_{x_{r}}
$$

Set $P_{i}=P_{x_{i}}, Q_{i}=Q_{x_{i}}, S_{i}=S_{x_{i}}$, and

$$
P=\sum_{i=1}^{r} S_{i}^{2} Q_{i} P_{i}, \quad Q=\sum_{i=1}^{r} S_{i}^{2} Q_{i}^{2} .
$$


Then $U=\mathbb{R}^{n} \backslash Z(Q)$ and $S_{i}^{2} Q_{i} f=S_{i}^{2} P_{i}$ on $X$. Consequently, $f=P / Q$ on $X$, hence (e) holds. The proof is complete.

A filtration of a real algebraic variety $V$ is a finite sequence $\mathscr{F}=\left(V_{0}, V_{1}, \ldots, V_{m+1}\right)$ of Zariski closed subvarieties of $V$ satisfying

$$
V=V_{0} \supseteq V_{1} \supseteq \cdots \supseteq V_{m+1}=\varnothing
$$

We allow $V_{i}=V_{i+1}$ for some $i$. Note that $\overline{\mathscr{F}}:=\left\{V_{i} \backslash V_{i+1}: 0 \leq i \leq m\right\}$ is a stratification of $V$.

The following is a generalization of [61, Proposition 2.2].

Proposition 2.3 Let $V, W$ be real algebraic varieties, and $f: X \rightarrow W$ a map defined on some subset $X \subseteq V$. Then the following conditions are equivalent:

(a) There exists a stratification $\mathscr{S}$ of $V$ such that for every stratum $S \in \mathscr{S}$ the restriction of $f$ to $X \cap S$ is a regular map.

(b) There exists a filtration $\mathscr{F}$ of $V$ such that for every stratum $T \in \overline{\mathscr{F}}$ the restriction of $f$ to $X \cap T$ is a regular map.

(c) For every Zariski closed subvariety $Z \subseteq V$, there exists a Zariski open dense subset $Z^{0} \subseteq Z$ such that the restriction of $f$ to $X \cap Z^{0}$ is a regular map.

In particular, the map $f$ is stratified-regular if and only if it is continuous and satisfies the equivalent conditions (a), (b), (c).

Proof It is clear that (b) implies (a).

Suppose that (a) holds, and let $Z \subseteq V$ be an irreducible Zariski closed subvariety. We can find a stratum $S \in \mathscr{S}$ such that the intersection $S \cap Z$ is nonempty and Zariski open (hence Zariski dense) subset of $Z$. Thus (c) holds for each irreducible $Z$. It immediately follows that (c) holds in the general case.

Now suppose that (c) is satisfied. Set $V_{0}=V$. Making use of (c) with $Z=V_{0}$, we find a Zariski closed nowhere dense subvariety $V_{1} \subseteq V_{0}$ such that the restriction of $f$ to $X \cap\left(V_{0} \backslash V_{1}\right)$ is a regular map. Note that $\operatorname{dim} V_{1}<\operatorname{dim} V_{0}$. We repeat this construction with $Z=V_{1}$ to get $V_{2} \subseteq V_{1}$, and so on. This process terminates after finitely many steps with $V_{m+1}=\varnothing$, which proves (b).

Let $V, W$ be real algebraic varieties, $X \subseteq V$ some subset, and $Z$ the Zariski closure of $X$ in $V$. We say that a map $f: X \rightarrow W$ is rational if there exists a Zariski open dense subset $Z^{0} \subseteq Z$ such that the restriction of $f$ to $X \cap Z^{0}$ is a regular map (no condition on the restriction of $f$ to $X \backslash\left(X \cap Z^{0}\right)$ is imposed).

In view of Proposition 2.3, each stratified-regular map is continuous rational. On the other hand, if the set $\operatorname{Sing}(V)$ of singular points of $V$ (that is, the complement in $V$ of the locus of all nonsingular points of $V$ ) is nonempty, then it can happen that a function from $V$ into $\mathbb{R}$ is continuous rational but it is not stratified-regular, cf. [45, Example 2]. However, the following holds.

Proposition 2.4 Let $V$, $W$ be real algebraic varieties, and $f: U \rightarrow W$ a map defined on an open subset $U \subseteq V \backslash \operatorname{Sing}(V)$. Then the following conditions are equivalent: 
(a) The map $f$ is stratified-regular.

(b) The map $f$ is continuous and rational.

Proof We may assume that $W \subseteq \mathbb{R}^{p}$ is a Zariski closed subvariety. Hence, by Lemma 2.1, the proof is reduced to the case $W=\mathbb{R}$, which follows from [44, Proposition 4.2] (a variant of [45, Proposition 8]).

It is worthwhile to make a comment on the terminology used in different papers.

Remark 2.5 In view of Proposition 2.4, reading papers [50,52,54,55,58,60] one can substitute everywhere stratified-regular maps for continuous rational maps. It follows from Proposition 2.3 that stratified-regular functions coincide with continuous hereditarily rational functions studied in [44,45]. Furthermore, as explained in [29,61,66], stratified-regular maps defined on a constructible subset of a real algebraic variety are identical with regulous maps.

We now present a counterpart of Proposition 2.3 for piecewise-regular maps.

Proposition 2.6 Let $V, W$ be real algebraic varieties, and $f: X \rightarrow W$ a map defined on some subset $X \subseteq V$. Then the following conditions are equivalent:

(a) There exists a stratification $\mathscr{S}$ of $V$ such that for every stratum $S \in \mathscr{S}$ the restriction of $f$ to each connected component of $X \cap S$ is a regular map.

(b) There exists a filtration $\mathscr{F}$ of $V$ such that for every stratum $T \in \overline{\mathscr{F}}$ the restriction of $f$ to each connected component of $X \cap T$ is a regular map.

(c) For every Zariski closed subvariety $Z \subseteq V$ there exists a Zariski open dense subset $Z^{0} \subseteq Z$ such that the restriction of $f$ to each connected component of $X \cap Z^{0}$ is a regular map.

In particular, the map $f$ is piecewise-regular if and only if it is continuous and satisfies the equivalent conditions (a), (b), (c).

Proof One can repeat the proof of Proposition 2.3 with only minor modifications.

We also have the following characterization of piecewise-regular maps.

Proposition 2.7 Let $V, W$ be real algebraic varieties, and $f: X \rightarrow W$ a continuous map defined on some subset $X \subseteq V$. Then the following conditions are equivalent:

(a) The map $f$ is piecewise-regular.

(b) There exists a stratification $\mathscr{S}$ of $V$ such that for every stratum $S \in \mathscr{S}$ the restriction $\left.f\right|_{X \cap S}: X \cap S \rightarrow W$ is a piecewise-regular map.

Proof It is clear that (a) implies (b).

Suppose that (b) holds for some stratification $\mathscr{S}$ of $V$. For each stratum $S \in \mathscr{S}$ there exists a stratification $\mathscr{T}_{S}$ of $V$ such that for every stratum $T \in \mathscr{T}_{S}$ the restriction of $f$ to each connected component of $X \cap S \cap T$ is a regular map. Note that

$$
\mathscr{P}:=\left\{S \cap T: S \in \mathscr{S} \text { and } T \in \mathscr{T}_{S}\right\}
$$

is a stratification of $V$, and the map $f$ is piecewise $\mathscr{P}$-regular. Thus (b) implies (a), as required. 
Given a real algebraic variety $V$, a subset $A \subseteq V$ is said to be a nonsingular algebraic arc if its Zariski closure $C$ in $V$ is an algebraic curve (that is, $\operatorname{dim} C=1$ ), $A \subseteq C \backslash \operatorname{Sing}(C)$, and $A$ is homeomorphic to $\mathbb{R}$.

Proposition 2.8 Let $V, W$ be real algebraic varieties, and $f: X \rightarrow W$ a continuous map defined on a semialgebraic subset $X \subseteq V$. Then the following conditions are equivalent:

(a) The map $f$ is piecewise-regular.

(b) There exists a stratification $\mathscr{S}$ of $V$ such that for every stratum $S \in \mathscr{S}$ and every nonsingular algebraic arc $A$ in $V$, with $A \subseteq X \cap S$, the restriction $\left.f\right|_{A}$ is a regular map.

Proof We may assume that $W \subseteq \mathbb{R}^{p}$ is a Zariski closed subvariety. Hence, by Lemma 2.1, the proof is reduced to the case $W=\mathbb{R}$.

It is clear that (a) implies (b).

Suppose that (b) holds for some stratification $\mathscr{S}$ of $V$. In view of [44, Proposition 3.5], for every stratum $S \in \mathscr{S}$, the restriction $\left.f\right|_{X \cap S}$ is a piecewise-regular map. Consequently, by Proposition 2.7, $f$ is a piecewise-regular map. Hence (b) implies (a), as required.

Piecewise-regular maps can be characterized among semialgebraic maps as follows.

Theorem 2.9 Let $V, W$ be real algebraic varieties, $X \subseteq V$ a semialgebraic subset, and $f: X \rightarrow W$ a continuous semialgebraic map. Then the following conditions are equivalent:

(a) The map $f$ is piecewise-regular.

(b) For every nonsingular algebraic arc $A$ in $V$, with $A \subseteq X$, the restriction $\left.f\right|_{A}$ is a piecewise-regular map.

(c) For every nonsingular algebraic arc $A$ in $V$, with $A \subseteq X$, there exists a nonempty open subset $A_{0} \subseteq A$ such that the restriction $\left.f\right|_{A_{0}}$ is a regular map.

Proof As in the proof of Proposition 2.8, we may assume that $W=\mathbb{R}$.

Evidently, (a) implies (b), and (b) implies (c).

Suppose that (c) holds, and let $Z \subseteq V$ be a Zariski closed subvariety. Let $Y$ be the Zariski closure of $X \cap Z$ in $V$. By Lemma 2.11 below (with $X$ replaced by $X \cap Z$ ), there exists a Zariski open dense subset $Y^{0} \subseteq Y$ such that the restriction of $f$ to each connected component of $(X \cap Z) \cap Y^{0}$ is a regular function. Note that $Z^{0}:=Z \backslash\left(Y \backslash Y^{0}\right)$ is a Zariski open dense subset of $Z$, and

$$
(X \cap Z) \cap Y^{0}=X \cap Z^{0} .
$$

Hence, in view of Proposition 2.6, condition (a) holds.

For background on Nash manifolds and Nash functions we refer to [6]. The following variant of [44, Propositon 2.5] will be useful in the proof of Lemma 2.11. 
Lemma 2.10 Let $N \subseteq \mathbb{R}^{n}$ be a connected Nash submanifold, and $f: N \rightarrow \mathbb{R}$ a Nash function. Assume that for every nonsingular algebraic arc $A$ in $\mathbb{R}^{n}$, with $A \subseteq N$, there exists a nonempty open subset $A_{0} \subseteq A$ such that the restriction $\left.f\right|_{A_{0}}$ is a regular function. Then $f$ is a rational function.

Proof Let $V$ be the Zariski closure of $N$ in $\mathbb{R}^{n}$. Note that $V$ is irreducible. Furthermore, the Zariski closure of the graph of $f$ in $V \times \mathbb{R}$ is also irreducible. Complexifying these data, we complete the proof arguing as in [44, Proposition 2.5].

In the proof of Theorem 2.9 we used the following.

Lemma 2.11 Let $V$ be a real algebraic variety, $X \subseteq V$ a semialgebraic set, and $f: X \rightarrow \mathbb{R}$ a semialgebraic function. Let $Y$ be the Zariski closure of $X$ in $V$. Assume that for every nonsingular algebraic arc $A$ in $V$, with $A \subseteq X$, there exists a nonempty open subset $A_{0} \subseteq A$ such that the restriction $\left.f\right|_{A_{0}}$ is a regular function. Then there exists a Zariski open dense subset $Y^{0} \subseteq Y$ such that the restriction of $f$ to each connected component of $X \cap Y^{0}$ is a regular function.

Proof We may assume that $V \subseteq \mathbb{R}^{n}$ is a Zariski closed subvariety. Set $Y_{0}=$ $Y \backslash \operatorname{Sing}(Y)$, and let $X^{*}$ be the interior of $X \cap Y_{0}$ in $Y_{0}$. Then $X \backslash X^{*}$ is a semialgebraic subset of $Y$ whose Zariski closure is Zariski nowhere dense in $Y$, cf. [6, Chapter 2]. Clearly, each connected component of $X^{*}$ is a Nash manifold. Therefore, there exists a Zariski closed and Zariski nowhere dense subvariety $S \subseteq Y$ such that

$$
\left(X \backslash X^{*}\right) \cup \operatorname{Sing}(Y) \subseteq S
$$

and the restriction of $f$ to each connected component of $X \backslash S$ is a Nash function, cf. for example [28, (2.4.1)]. Since $X \backslash S$ is a semialgebraic set, it has finitely many connected components. Hence, in view of Lemma 2.10, there exists a Zariski open dense subset $Y^{0} \subseteq Y$ which has the required properties.

We next deal with piecewise-regular maps of class $\mathscr{C}^{\infty}$. Initially, we consider functions on nonsingular real algebraic arcs.

Lemma 2.12 Let $C$ be a real algebraic curve, $A \subseteq C \backslash \operatorname{Sing}(C)$ a nonsingular real algebraic arc, and $f: A \rightarrow \mathbb{R}$ a piecewise-regular function of class $\mathscr{C}^{\infty}$. Then $f$ is a regular function.

Proof The function $f$ is analytic, being semialgebraic and of class $\mathscr{C}^{\infty}$, cf. [6, Proposition 8.1.8].

By definition of piecewise-regular map, we can find a Zariski open dense subset $C^{0} \subseteq C \backslash \operatorname{Sing}(C)$, a regular function $\varphi: C^{0} \rightarrow \mathbb{R}$ and a nonempty open subset $U \subseteq A \cap C^{0}$ such that $\left.f\right|_{U}=\left.\varphi\right|_{U}$. Regarding $\mathbb{R}$ as a subset of $\mathbb{P}^{1}(\mathbb{R})$, we get a regular map $\psi: C \backslash \operatorname{Sing}(C) \rightarrow \mathbb{P}^{1}(\mathbb{R})$ with $\left.\psi\right|_{C^{0}}=\varphi$. Hence $f=\left.\psi\right|_{A}$ by the identity principle for analytic maps. Consequently, $f$ is a regular function.

Let $V, W$ be real algebraic varieties and let $A \subseteq V, B \subseteq W$ be arbitrary subsets. We say that a map $g: A \rightarrow B$ is of class $\mathscr{C}^{\infty}$ if for some algebraic embeddings 
$\varphi: V \rightarrow \mathbb{R}^{n}, \psi: W \rightarrow \mathbb{R}^{p}$ there exist an open neighborhood $U \subseteq \mathbb{R}^{n}$ of $\varphi(A)$ and a $\mathscr{C}^{\infty}$ map $\tilde{g}: U \rightarrow \mathbb{R}^{p}$ such that $\tilde{g}(\varphi(x))=\psi(g(x))$ for all $x \in \bar{A}$. If this holds, then the same condition holds for any choice of the algebraic embeddings $\varphi$ and $\psi$.

Lemma 2.12 can be generalized as follows.

Proposition 2.13 Let $V, W$ be real algebraic varieties, and $f: U \rightarrow W$ a map defined on an open subset $U \subseteq V \backslash \operatorname{Sing}(V)$. Then the following conditions are equivalent:

(a) The map $f$ is piecewise-regular and of class $\mathscr{C}^{\infty}$.

(b) The restriction of $f$ to each connected component of $U$ is a regular map.

Proof As in the proof of Proposition 2.8, we may assume that $W=\mathbb{R}$.

Suppose that (a) holds and let $U_{0}$ be a connected component of $U$. For each nonsingular algebraic arc $A$ in $V$, with $A \subseteq U_{0}$, the restriction $\left.f\right|_{A}$ is a regular function by Lemma 2.12. Hence, in view of [44, Theorem 2.4], $\left.f\right|_{U_{0}}$ is a rational function. Consequently, $\left.f\right|_{U_{0}}$ is a regular function according to [50, Proposition 2.1].

It is clear that (b) implies (a).

We also have the following variant of Proposition 2.13.

Proposition 2.14 Let $V, W$ be real algebraic varieties, and $f: U \rightarrow W$ a map defined on an open subset $U \subseteq V \backslash \operatorname{Sing}(V)$. Then the following conditions are equivalent:

(a) The map $f$ is piecewise-regular, and for every nonsingular algebraic arc $A$ in $V$, with $A \subseteq U$, the restriction $\left.f\right|_{A}$ is of class $\mathscr{C}^{\infty}$.

(b) The restriction of $f$ to each connected component of $U$ is a stratified-regular map.

Proof As in the proof of Proposition 2.8, we may assume that $W=\mathbb{R}$. It suffices to consider the case where $U$ is connected.

Suppose that (a) holds. For each nonsingular algebraic arc $A$ in $V$, with $A \subseteq U$, the restriction $\left.f\right|_{A}$ is a regular function by Proposition 2.13. Hence, in view of [44, Theorem 2.4], $f$ is a rational function. Consequently, $f$ is a stratified-regular function according to Proposition 2.4.

It is clear that (b) implies (a).

\section{Functions on a simplex}

This section is of a technical nature. Our main goal is Lemma 3.7, which is needed in Sects. 4 and 6. For the sake of clarity, we begin with some preliminary facts.

Lemma 3.1 Let $V$ be a real algebraic variety, $W \subseteq V$ a Zariski closed subvariety, and $f: W \rightarrow \mathbb{R}$ a regular function. Then there exists a regular function $F: V \rightarrow \mathbb{R}$ such that $\left.F\right|_{W}=f$.

Proof By Lemma 2.1, there exist regular functions $\varphi, \psi: V \rightarrow \mathbb{R}$ such that $W \subseteq$ $V \backslash Z(\psi)$ and $f=\varphi / \psi$ on $W$. Pick a regular function $\alpha: V \rightarrow \mathbb{R}$ with $Z(\alpha)=W$. Then the function $F:=\frac{\varphi \psi}{\alpha^{2}+\psi^{2}}$ has the required properties. 
For any real algebraic variety $V$, we let $\mathscr{R}(V)$ denote the ring of real-valued regular functions on $V$. If $W \subseteq V$ is a Zariski closed subvariety, then the ideal

$$
I_{V}(W)=\left\{f \in \mathscr{R}(V):\left.f\right|_{W}=0\right\}
$$

of $\mathscr{R}(V)$ is called the ideal of $W$ in $V$.

Lemma 3.2 Let $V$ be a real algebraic variety, and $W_{1}, W_{2}$ Zariski closed subvarieties of $V$ for which

$$
I_{V}\left(W_{1} \cap W_{2}\right)=I_{V}\left(W_{1}\right)+I_{V}\left(W_{2}\right) \text { in } \mathscr{R}(V) .
$$

Let $f: W_{1} \cup W_{2} \rightarrow \mathbb{R}$ be a function such that the restrictions $\left.f\right|_{W_{1}}$ and $\left.f\right|_{W_{2}}$ are regular functions. Then $f$ is a regular function.

Proof By Lemma 3.1, there exists a regular function $g_{i}: V \rightarrow \mathbb{R}$ with $\left.g_{i}\right|_{W_{i}}=\left.f\right|_{W_{i}}$ for $i=1$, 2. Since $g_{1}-g_{2} \in I_{V}\left(W_{1} \cap W_{2}\right)$, we have $g_{1}-g_{2}=h_{1}-h_{2}$, where $h_{i} \in I_{V}\left(W_{i}\right)$. Hence $g_{1}-h_{1}=g_{2}-h_{2}$ is a regular function on $V$ whose restriction to $W_{1} \cup W_{2}$ is equal to $f$. Consequently, $f$ is a regular function.

Let $V$ be a nonsingular real algebraic variety, and $D \subseteq V$ a Zariski closed subvariety. We say that $D$ is a simple normal crossing hypersurface if for each point $p \in D$ there exist a Zariski open neighborhood $U \subseteq V$ of $p$ and local coordinates $x_{1}, \ldots, x_{n}$ on $U$ (a regular system of parameters at $p \in V$ ) such that the intersection of each irreducible component of $D$ with $U$ is given by the equation $x_{i}=0$ for a suitable $i$. In particular, if $D$ is a simple normal crossing hypersurface, then each irreducible component of $D$ is nonsingular of codimension 1 .

Lemma 3.3 Let $V$ be a nonsingular real algebraic variety, $D \subseteq V$ a simple normal crossing hypersurface, and $f: D \rightarrow \mathbb{R}$ a function whose restriction to each irreducible component of $D$ is a regular function. Then $f$ is a regular function.

Proof We use induction on the number $k$ of irreducible components of $D$. The case $k=1$ is obvious. Suppose that $k \geq 2$. Let $D_{1}$ be an irreducible component of $D$, and let $D^{\prime}$ be the union of the remaining irreducible components. The restriction $\left.f\right|_{D_{1}}$ is a regular function by assumption, whereas the restriction $\left.f\right|_{D^{\prime}}$ is a regular function by the induction hypothesis.

Pick a point $p \in D_{1} \cap D^{\prime}$. It suffices to find a Zariski open neighborhood $U \subseteq V$ of $p$ such that $\left.f\right|_{D \cap U}$ is a regular function. If $U$ is small enough, there exist local coordinates $x_{1}, \ldots, x_{n}$ on $U$ such that the ideal $I_{U}\left(D_{1} \cap U\right)$ is generated by $x_{1}$, and the ideal $I_{U}\left(D^{\prime} \cap U\right)$ is generated by the product $x_{2} \cdots x_{l}$ for some $l$ with $2 \leq l \leq n$. Note that the ideal $I_{U}\left(D_{1} \cap D^{\prime} \cap U\right)$ is generated by $x_{1}$ and $x_{2} \cdots x_{l}$; in other words,

$$
I_{U}\left(D_{1} \cap D^{\prime} \cap U\right)=I_{U}\left(D_{1} \cap U\right)+I_{U}\left(D^{\prime} \cap U\right) \text { in } \mathscr{R}(U) .
$$

Hence $\left.f\right|_{D \cap U}$ is a regular function in view of Lemma 3.2.

We give next the following variant of Lemma 3.1. 
Lemma 3.4 Let $V$ be a real algebraic variety, $Y \subseteq X$ some subsets of $V$, and $f: Y \rightarrow$ $\mathbb{R}$ a regular function. Assume that $Y=X \cap W$, where $W$ is the Zariski closure of $Y$ in $V$. Then there exists a regular function $F: V_{0} \rightarrow \mathbb{R}$, defined on a Zariski open neighborhood $V_{0}$ of $X$ in $V$, such that $\left.F\right|_{Y}=f$.

Proof By Lemma 2.2, there exist regular functions $\varphi, \psi: V \rightarrow \mathbb{R}$ such that $Y \subseteq V \backslash Z(\psi)$ and $f=\varphi / \psi$ on $Y$. Pick a regular function $\alpha: V \rightarrow \mathbb{R}$ with $Z(\alpha)=W$. Set $V_{0}:=V \backslash Z\left(\alpha^{2}+\psi^{2}\right)$ and $F:=\frac{\varphi \psi}{\alpha^{2}+\psi^{2}}$ on $V_{0}$. Then $F$ has the required properties.

Notation 3.5 By a simplex in $\mathbb{R}^{m}$ we always mean a closed geometric simplex. For any finite (geometric) simplicial complex $K$ in $\mathbb{R}^{m}$, we write $|K|$ for the union of all simplices in $K$; thus $|K| \subseteq \mathbb{R}^{m}$ is a compact polyhedron. We denote by $K^{(n)}$ the $n$-skeleton of $K$.

If $\Delta \subseteq \mathbb{R}^{m}$ is a $d$-simplex, then $\dot{\Delta}$ stands for the simplicial complex which consists of all faces of $\Delta$ of dimension at most $d-1$. Clearly, $|\dot{\Delta}|$ is the union of all $(d-1)$ dimensional faces of $\Delta$. The Zariski closure of $\Delta$ in $\mathbb{R}^{m}$, denoted by $H_{\Delta}$, is an affine subspace of dimension $d$.

Lemma 3.6 Let $\Delta \subseteq \mathbb{R}^{m}$ be a d-simplex and let $f:|\dot{\Delta}| \rightarrow \mathbb{R}$ be a function such that the restriction $\left.f\right|_{\Gamma}: \Gamma \rightarrow \mathbb{R}$ is a regular function for every $(d-1)$-simplex $\Gamma \in \dot{\Delta}$. Then there exists a regular function $F: \Delta \rightarrow \mathbb{R}$ with $\left.F\right|_{|\dot{\Delta}|}=f$.

Proof Let $\Delta_{0}, \ldots, \Delta_{d}$ be all the $(d-1)$-dimensional faces of $\Delta$. We set $H:=H_{\Delta}$ and $H_{i}:=H_{\Delta_{i}}$ for $i=0, \ldots, d$. Obviously, $H_{i} \subseteq H$ and $\Delta_{i}=\Delta \cap H_{i}$. By Lemma 3.4, there exists a Zariski open neighborhood $\Omega \subseteq H$ of $\Delta$ and a regular function $G_{i}: \Omega \rightarrow \mathbb{R}$ with $\left.G_{i}\right|_{\Delta_{i}}=\left.f\right|_{\Delta_{i}}$ for $i=0, \ldots, d$. In particular, $G_{i}=G_{j}$ on $\Delta_{i} \cap \Delta_{j}$ for all $i, j$. Since $H_{i} \cap H_{j}$ is the Zariski closure of $\Delta_{i} \cap \Delta_{j}$ in $\mathbb{R}^{m}$, we get $G_{i}=G_{j}$ on $\Omega \cap H_{i} \cap H_{j}$.

Set $D_{i}:=\Omega \cap H_{i}$ and $D:=D_{0} \cup \cdots \cup D_{d}$. Then $D$ is a simple normal crossing hypersurface in $\Omega$. Define a function $\varphi: D \rightarrow \mathbb{R}$ by $\left.\varphi\right|_{D_{i}}=\left.G_{i}\right|_{D_{i}}$ for $i=0, \ldots, d$. By Lemma 3.3, $\varphi$ is a regular function. In view of Lemma 3.1, there exists a regular function $\Phi: \Omega \rightarrow \mathbb{R}$ with $\left.\Phi\right|_{D}=\varphi$. The function $F:=\left.\Phi\right|_{\Delta}$ has the required properties.

We need the following approximation result for functions defined on a simplex.

Lemma 3.7 Let $\Delta \subseteq \mathbb{R}^{m}$ be a d-simplex and let $f: \Delta \rightarrow \mathbb{R}$ be a continuous function such that the restriction $\left.f\right|_{\Gamma}: \Gamma \rightarrow \mathbb{R}$ is a regular function for every $(d-1)$-simplex $\Gamma \in \dot{\Delta}$. Then, for every $\varepsilon>0$, there exists a regular function $g: \Delta \rightarrow \mathbb{R}$ satisfying

$$
|f(x)-g(x)|<\varepsilon \text { for all } x \in \Delta
$$

and $\left.f\right|_{|\dot{\Delta}|}=\left.g\right|_{|\dot{\Delta}|}$.

Proof According to Lemma 3.6, there exists a regular function $h: \Delta \rightarrow \mathbb{R}$ with $\left.h\right|_{|\dot{\Delta}|}=\left.f\right|_{|\dot{\Delta}|}$. By replacing $f$ with $f-h$, the proof is reduced to the case $\left.f\right|_{|\dot{\Delta}|}=0$. 
Let $\Delta_{0}, \ldots, \Delta_{d}$ be all the $(d-1)$-dimensional faces of $\Delta$. We set $H:=H_{\Delta}$ and $H_{i}:=H_{\Delta_{i}}$ for $i=0, \ldots, d$. The union $D:=H_{0} \cup \cdots \cup H_{d}$ is a simple normal crossing hypersurface in $H$. The function on $\Delta \cup D$ which is equal to $f$ on $\Delta$ and identically equal to 0 on $D$ is continuous. Hence, by Tietze's extension theorem, there exists a continuous function $\varphi: H \rightarrow \mathbb{R}$ with $\left.\varphi\right|_{\Delta}=f$ and $\left.\varphi\right|_{D}=0$.

Fix $\varepsilon>0$. Note that there exists a $\mathscr{C}^{\infty}$ function $\psi: H \rightarrow \mathbb{R}$ satisfying

$$
|\varphi(x)-\psi(x)|<\frac{\varepsilon}{2} \text { for all } x \in H
$$

and $\left.\psi\right|_{D}=0$. Indeed, by the Whitney approximation theorem [67, Theorem 10.16], one can find a $\mathscr{C}^{\infty}$ function $\lambda: H \rightarrow \mathbb{R}$ for which

$$
|\varphi(x)-\lambda(x)|<\frac{\varepsilon}{4} \text { for all } x \in H
$$

Since $\left.\varphi\right|_{D}=0$, the set $U:=\left\{x \in H:|\lambda(x)|<\frac{\varepsilon}{4}\right\}$ is an open neighborhood of $D$ in $H$. If $\alpha: H \rightarrow \mathbb{R}$ is a $\mathscr{C}^{\infty}$ function with $\left.\alpha\right|_{D}=1,0 \leq \alpha \leq 1$ on $H$ and support contained in $U$, then the function $\psi:=(1-\alpha) \lambda$ has the required properties.

Denote by $\mathscr{C}^{\infty}(H)$ the ring of $\mathscr{C}^{\infty}$ real-valued functions on $H$. One readily sees that the ideal $I \subseteq \mathscr{C}^{\infty}(H)$ of all $\mathscr{C}^{\infty}$ functions vanishing on $D$ is generated by polynomial functions, say, $q_{1}, \ldots, q_{r}$ (alternatively, one can invoke a much more general result [81, p. 52, Proposition 1]). Consequently, $\psi$ can be written in the form

$$
\psi=\psi_{1} q_{1}+\cdots+\psi_{r} q_{r}
$$

where the $\psi_{k}$ are $\mathscr{C}^{\infty}$ functions on $H$. Let

$$
M:=\sup \left\{\left|q_{k}(x)\right|: x \in \Delta, k=1, \ldots, r\right\} .
$$

By the Weierstrass approximation theorem, there exists a polynomial function $p_{k}: H \rightarrow \mathbb{R}$ satisfying

$$
\left|\psi_{k}(x)-p_{k}(x)\right|<\frac{\varepsilon}{2 r M} \text { for all } x \in \Delta .
$$

For $p:=p_{1} q_{1}+\cdots+p_{r} q_{r}$, we have

$$
|\varphi(x)-p(x)| \leq|\varphi(x)-\psi(x)|+|\psi(x)-p(x)|<\frac{\varepsilon}{2}+\frac{\varepsilon}{2}=\varepsilon \text { for all } x \in \Delta
$$

and $\left.p\right|_{D}=0$. We complete the proof setting $g:=\left.p\right|_{\Delta}$.

\section{Piecewise-regular maps into Grassmannians}

The role of Sects. 4.1 and 4.2 is to review some notation and terminology. 


\subsection{Inner product and matrices}

As in Sect. 1, we let $\mathbb{F}$ denote $\mathbb{R}, \mathbb{C}$ or $\mathbb{H}$. The $\mathbb{F}$-vector space $\mathbb{F}^{n}$ is endowed with the standard inner product

$$
\langle-,-\rangle: \mathbb{F}^{n} \times \mathbb{F}^{n} \rightarrow \mathbb{F}
$$

given by

$$
\left\langle\left(x_{1}, \ldots, x_{n}\right),\left(y_{1}, \ldots, y_{n}\right)\right\rangle=\sum_{i=1}^{n} x_{i} \overline{y_{i}},
$$

where $\overline{y_{i}}$ stands for the conjugate of $y_{i}$ in $\mathbb{F}$.

Let $\mathrm{Mat}_{m, n}(\mathbb{F})$, or simply $\mathrm{Mat}_{n}(\mathbb{F})$ if $m=n$, denote the set of all $m$-by- $n$ matrices with entries in $\mathbb{F}$. For any matrix $A=\left[a_{i j}\right] \in \mathrm{Mat}_{m, n}(\mathbb{F})$, the corresponding $\mathbb{F}$-linear transformation $L_{A}: \mathbb{F}^{n} \rightarrow \mathbb{F}^{m}$ is given by

$$
\left(x_{1}, \ldots, x_{n}\right) \mapsto\left(y_{1}, \ldots, y_{m}\right), \quad \text { where } y_{i}=\sum_{j=1}^{n} x_{j} a_{i j} \text { for } i=1, \ldots, m
$$

(recall that we always consider left $\mathbb{F}$-vector spaces). We will identify $A$ with $L_{A}$ and write

$$
A(v)=L_{A}(v) \text { for } v \in \mathbb{F}^{n} .
$$

If $B=\left[b_{j k}\right] \in \operatorname{Mat}_{n, r}(\mathbb{F})$, then we define the product $A B=\left[c_{i k}\right]$ by

$$
c_{i k}=\sum_{j=1}^{n} b_{j k} a_{i j}
$$

This convention implies that $L_{A B}=L_{A} \circ L_{B}$.

We regard $\operatorname{Mat}_{n, r}(\mathbb{F})$ as a real algebraic variety. If $1 \leq r \leq n$, then the subset

$$
\operatorname{Mat}_{n, r}^{0}(\mathbb{F}) \subseteq \operatorname{Mat}_{n, r}(\mathbb{F})
$$

of all matrices with linearly independent columns is Zariski open. Furthermore, the map

$$
\operatorname{Mat}_{n, r}^{0}(\mathbb{F}) \rightarrow \mathbb{G}_{r}\left(\mathbb{F}^{n}\right), \quad A \mapsto A\left(\mathbb{F}^{r}\right)
$$

is a regular map, as is immediately seen by using the standard charts on $\mathbb{G}_{r}\left(\mathbb{F}^{n}\right)$.

\subsection{Vector bundles}

For any topological $\mathbb{F}$-vector bundle $\xi$ on a topological space $X$, we denote by $E(\xi)$ its total space and by $p(\xi): E(\xi) \rightarrow X$ the bundle projection. The fiber of $\xi$ over a point $x \in X$ is $E(\xi)_{x}=p(\xi)^{-1}(x)$.

Given a nonnegative integer $n$, we let $\varepsilon_{X}^{n}(\mathbb{F})$ denote the standard product $\mathbb{F}$-vector bundle on $X$ with total space $X \times \mathbb{F}^{n}$. Any morphism $\varphi: \varepsilon_{X}^{r}(\mathbb{F}) \rightarrow \varepsilon_{X}^{n}(\mathbb{F})$ of topological 
$\mathbb{F}$-vector bundles is of the form

$$
\varphi(x, v)=\left(x, A_{\varphi}(x)(v)\right) \text { for all }(x, v) \in X \times \mathbb{F}^{r},
$$

where $A_{\varphi}: X \rightarrow \operatorname{Mat}_{n, r}(\mathbb{F})$ is a uniquely determined map, called the matrix representation of $\varphi$. Obviously, $A_{\varphi}$ is a continuous map.

If $\xi$ is a topological $\mathbb{F}$-vector subbundle of $\varepsilon_{X}^{n}(\mathbb{F})$, then $\varepsilon_{X}^{n}(\mathbb{F})=\xi \oplus \xi^{\perp}$, where $\xi^{\perp}$ is the orthogonal complement of $\xi$ with respect to the standard inner product on $\mathbb{F}^{n}$. The orthogonal projection $\rho_{\xi}: \varepsilon_{X}^{n}(\mathbb{F}) \rightarrow \varepsilon_{X}^{n}(\mathbb{F})$ onto $\xi$ is a topological morphism of $\mathbb{F}$-vector bundles.

We will also consider algebraic vector bundles on a real algebraic variety $V$. The product $V \times \mathbb{F}^{n}$ will be regarded as a real algebraic variety. By an algebraic $\mathbb{F}$-vector bundle on $V$ we mean an algebraic $\mathbb{F}$-vector subbundle of $\varepsilon_{V}^{n}(\mathbb{F})$ for some $n$ (cf. [6, Chapters 12 and 13] and [37,38] for various characterizations of algebraic $\mathbb{F}$-vector bundles).

If $\varphi: \varepsilon_{V}^{r}(\mathbb{F}) \rightarrow \varepsilon_{V}^{n}(\mathbb{F})$ is an algebraic morphism, then the matrix representation

$$
A_{\varphi}: V \rightarrow \operatorname{Mat}_{n, r}(\mathbb{F})
$$

of $\varphi$ is a regular map.

If $\xi$ is an algebraic $\mathbb{F}$-vector subbundle of $\varepsilon_{V}^{n}(\mathbb{F})$, then its orthogonal complement $\xi^{\perp}$ is also an algebraic $\mathbb{F}$-vector subbundle, and the orthogonal projection $\rho_{\xi}: \varepsilon_{V}^{n}(\mathbb{F}) \rightarrow$ $\varepsilon_{V}^{n}(\mathbb{F})$ onto $\xi$ is an algebraic morphism.

The tautological $\mathbb{F}$-vector bundle $\gamma_{r}\left(\mathbb{F}^{n}\right)$ on $\mathbb{G}_{r}\left(\mathbb{F}^{n}\right)$ is an algebraic $\mathbb{F}$-vector subbundle of $\varepsilon_{\mathbb{G}_{r}(\mathbb{F})}^{n}\left(\mathbb{F}^{n}\right)$.

Lemma 4.1 Let $V$ be a real algebraic variety, and $f: X \rightarrow \mathbb{G}_{r}\left(\mathbb{F}^{n}\right)$ a continuous map defined on some subset $X \subseteq V$. Then the map $P_{f}: X \rightarrow \operatorname{Mat}_{n}(\mathbb{F})$, where $P_{f}(x): \mathbb{F}^{n} \rightarrow \mathbb{F}^{n}$ is the orthogonal projection onto $f(x) \subseteq \mathbb{F}^{n}$ for all $x \in X$, is continuous. Furthermore, if the map $f$ is regular, then so is the map $P_{f}$.

Proof We regard the pullback $\xi:=f^{*} \gamma_{r}\left(\mathbb{F}^{n}\right)$ as a topological $\mathbb{F}$-vector subbundle of $\varepsilon_{X}^{n}(\mathbb{F})$, hence

$$
E(\xi)_{x}=\{x\} \times f(x) \text { for all } x \in X .
$$

It follows that $P_{f}$ is the matrix representation of the orthogonal projection $\rho_{\xi}: \varepsilon_{X}^{n}(\mathbb{F}) \rightarrow \varepsilon_{X}^{n}(\mathbb{F})$ onto $\xi$. Consequently, $P_{f}$ is a continuous map.

Now, suppose that $f$ is a regular map. It suffices to consider the case where $X$ is a Zariski locally closed subvariety of $V$. Then $\xi$ is an algebraic $\mathbb{F}$-vector subbundle of $\varepsilon_{X}^{n}(\mathbb{F})$, hence the argument above shows that $P_{f}$ is a regular map.

\subsection{Maps into Grassmannians}

We can now prove the following variant of Lemma 3.7. 
Lemma 4.2 Let $\Delta \subseteq \mathbb{R}^{m}$ be a d-simplex and let $f: \Delta \rightarrow \mathbb{G}_{r}\left(\mathbb{F}^{n}\right)$ be a continuous map such that the restriction $\left.f\right|_{\Gamma}: \Gamma \rightarrow \mathbb{G}_{r}\left(\mathbb{F}^{n}\right)$ is a regular map for every $(d-1)$ simplex $\Gamma \in \dot{\Delta}$. Then, for each neighborhood $\mathscr{U} \subseteq \mathscr{C}\left(\Delta, \mathbb{G}_{r}\left(\mathbb{F}^{n}\right)\right)$ of $f$, there exists a regular map $g: \Delta \rightarrow \mathbb{G}_{r}\left(\mathbb{F}^{n}\right)$ such that $g \in \mathscr{U}$ and $\left.g\right|_{|\dot{\Delta}|}=\left.f\right|_{|\dot{\Delta}|}$.

Proof Consider the map $P=P_{f}: \Delta \rightarrow \operatorname{Mat}_{n}(\mathbb{F})$, where $P(x): \mathbb{F}^{n} \rightarrow \mathbb{F}^{n}$ is the orthogonal projection onto $f(x) \subseteq \mathbb{F}^{n}$ for all $x \in \Delta$. By Lemma 4.1, $P$ is a continuous map and the restriction $\left.P\right|_{\Gamma}: \Gamma \rightarrow \operatorname{Mat}_{n}(\mathbb{F})$ is a regular map for every $(d-1)$-simplex $\Gamma \in \dot{\Delta}$.

We regard the pullback $\xi:=f^{*} \gamma_{r}\left(\mathbb{F}^{n}\right)$ as a topological $\mathbb{F}$-vector subbundle of $\varepsilon_{\Delta}^{n}(\mathbb{F})$. Since $\xi$ is topologically trivial, there exists an injective topological morphism $\varphi: \varepsilon_{\Delta}^{r}(\mathbb{F}) \rightarrow \varepsilon_{\Delta}^{n}(\mathbb{F})$ whose image is equal to $\xi$. Let $A=A_{\varphi}: \Delta \rightarrow \operatorname{Mat}_{n, r}(\mathbb{F})$ be the matrix representation of $\varphi$. Then $A$ is a continuous map and

$$
P(x) A(x)=A(x) \text { for all } x \in \Delta .
$$

By the Weierstrass approximation theorem, there exists a regular map $B: \Delta \rightarrow \operatorname{Mat}_{n, r}(\mathbb{F})$ arbitrarily close to $A$. Define $C: \Delta \rightarrow \operatorname{Mat}_{n, r}(\mathbb{F})$ by

$$
C(x)=P(x) B(x) \text { for all } x \in \Delta \text {. }
$$

Then $C$ is a continuous map, close to $A$, such that the restriction $\left.C\right|_{\Gamma}: \Gamma \rightarrow$ $\operatorname{Mat}_{n, r}(\mathbb{F})$ is a regular map for every $(d-1)$-simplex $\Gamma \in \dot{\Delta}$. Hence, according to Lemma 3.7, there exists a regular map $\Phi: \Delta \rightarrow \operatorname{Mat}_{n, r}(\mathbb{F})$, arbitrarily close to $C$, with $\left.\Phi\right|_{|\dot{\Delta}|}=\left.C\right|_{|\dot{\Delta}|}$. In particular, the $\mathbb{F}$-linear transformation $\Phi(x): \mathbb{F}^{r} \rightarrow \mathbb{F}^{n}$ is injective for all $x \in \Delta$. In other words, $\Phi(\Delta) \subseteq \operatorname{Mat}_{n, r}^{0}(\mathbb{F})$. Thus

$$
g: \Delta \rightarrow \mathbb{G}_{r}\left(\mathbb{F}^{n}\right), \quad g(x)=\Phi(x)\left(\mathbb{F}^{r}\right)
$$

is a well-defined regular map, close to $f$. We may assume that $g \in \mathscr{U}$. Furthermore, $\left.g\right|_{|\dot{\Delta}|}=\left.f\right|_{|\dot{\Delta}|}$ since $\left.\Phi\right|_{|\dot{\Delta}|}=\left.C\right|_{|\dot{\Delta}|}$.

An important consequence of Lemma 4.2 is the following.

Proposition 4.3 Let $K$ be a finite simplicial complex in $\mathbb{R}^{m}$ and let $f:|K| \rightarrow \mathbb{G}\left(\mathbb{F}^{n}\right)$ be a continuous map. Then, for each open neighborhood $\mathscr{U} \subseteq \mathscr{C}\left(|K|, \mathbb{G}\left(\mathbb{F}^{n}\right)\right)$ of $f$, there exists a continuous map $\varphi:|K| \rightarrow \mathbb{G}\left(\mathbb{F}^{n}\right)$ such that $\varphi \in \mathscr{U}$ and the restriction $\left.\varphi\right|_{\Delta}: \Delta \rightarrow \mathbb{G}\left(\mathbb{F}^{n}\right)$ is a regular map for every simplex $\Delta \in K$.

Proof We use induction on $d=\operatorname{dim} K$. The case $d=0$ is obvious. Suppose now that $d \geq 1$. By the induction hypothesis, there exists a continuous map $\psi:\left|K^{(d-1)}\right| \rightarrow$ $\mathbb{G}\left(\mathbb{F}^{n}\right)$, arbitrarily close to $\left.f\right|_{\left|K^{(d-1)}\right|}$, such that the restriction $\left.\psi\right|_{\Gamma}: \Gamma \rightarrow \mathbb{G}\left(\mathbb{F}^{n}\right)$ is a regular map for every simplex $\Gamma \in K^{(d-1)}$.

We claim that $\psi$ has a continuous extension $\tilde{\psi}:|K| \rightarrow \mathbb{G}\left(\mathbb{F}^{n}\right)$ that belongs to $\mathscr{U}$. For the proof, we may assume that $\mathbb{G}\left(\mathbb{F}^{n}\right) \subseteq \mathbb{R}^{N}$ is a nonsingular Zariski closed subvariety. Regarding $f$ and $\psi$ as maps with values in $\mathbb{R}^{N}$, we have

$$
\|f(x)-\psi(x)\|<\varepsilon \text { for all } x \in\left|K^{(d-1)}\right|,
$$


where $\varepsilon>0$ is small and $\|-\|$ stands for the Euclidean norm on $\mathbb{R}^{N}$. According to Tietze's extension theorem, there exists a continuous map $\sigma:|K| \rightarrow \mathbb{R}^{N}$ with $\sigma_{\left|K^{(d-1)}\right|}=\psi$. If

$$
U:=\{x \in|K|:\|\sigma(x)-f(x)\|<\varepsilon\}
$$

and $\alpha:|K| \rightarrow \mathbb{R}$ is a continuous function with $\left.\alpha\right|_{\left|K^{(d-1)}\right|}=1,0 \leq \alpha \leq 1$ and support contained in $U$, then setting $h:=f+\alpha(\sigma-f)$, we get

$$
\|f(x)-h(x)\|=|\alpha(x)|\|\sigma(x)-f(x)\|<\varepsilon \text { for all } x \in|K| .
$$

Choose a tubular neighborhood $\rho: T \rightarrow \mathbb{G}\left(\mathbb{F}^{n}\right)$ of $\mathbb{G}\left(\mathbb{F}^{n}\right)$ in $\mathbb{R}^{N}$. If $\varepsilon$ is sufficiently small, then $h$ has values in $T$, and the composite $\tilde{\psi}=\rho \circ h$ has the properties required in the claim.

By Lemma 4.2, for every $d$-simplex $\Delta \in K$, there exists a regular map $g_{\Delta}: \Delta \rightarrow \mathbb{G}\left(\mathbb{F}^{n}\right)$ close to $\left.\tilde{\psi}\right|_{\Delta}$ and such that $\left.g_{\Delta}\right|_{|\dot{\Delta}|}=\left.\tilde{\psi}\right|_{|\dot{\Delta}|}$. Then the map $\varphi:|K| \rightarrow \mathbb{G}\left(\mathbb{F}^{n}\right)$, defined by $\left.\varphi\right|_{\left|K^{(d-1)}\right|}=\psi$ and $\left.\varphi\right|_{\Delta}=g_{\Delta}$ for every $d$-simplex $\Delta \in K$, has all the required properties.

In what follows, we will use stratifications constructed in a fairly simple way. Any finite simplicial complex $K$ in $\mathbb{R}^{m}$ gives rise to a filtration

$$
\mathscr{F}_{K}=\left(Z_{0}(K), Z_{1}(K), \ldots, Z_{m+1}(K)\right)
$$

of $\mathbb{R}^{m}$, where $Z_{0}(K)=\mathbb{R}^{m}, Z_{m+1}(K)=\varnothing$, and $Z_{d}(K)$ is the union of the $H_{\Delta}$ for all simplices $\Delta \in K$ of dimension at most $m-d$ with $d=1, \ldots, m$. Here, as in Notation 3.5, $H_{\Delta} \subseteq \mathbb{R}^{m}$ stands for the Zariski closure of $\Delta$. Setting

$$
\mathscr{S}_{K}:=\left\{Z_{i}(K) \backslash Z_{i+1}(K): i=0, \ldots, m\right\},
$$

we obtain a stratification of $\mathbb{R}^{m}$. More generally, if $V \subseteq \mathbb{R}^{m}$ is a Zariski closed subvariety, then the collection

$$
\mathscr{S}_{K}(V):=\left\{V \cap S: S \in \mathscr{S}_{K}\right\}
$$

is a stratification of $V$, which is said to be induced by $K$. Obviously,

$$
\mathscr{S}_{K}\left(\mathbb{R}^{m}\right)=\mathscr{S}_{K}
$$

Lemma 4.4 Let $K$ be a finite simplicial complex in $\mathbb{R}^{m}, V \subseteq \mathbb{R}^{m}$ a Zariski closed subvariety, and $X \subseteq|K| \cap V$ an arbitrary subset. Then, for every stratum $T \in \mathscr{S}_{K}(V)$, each connected component of $X \cap T$ is contained in some simplex $\Delta \in K$.

Proof It suffices to consider the case $V=\mathbb{R}^{m}$. If $S \in \mathscr{S}_{K}\left(\mathbb{R}^{m}\right)=\mathscr{S}_{K}$, then each connected component of $X \cap S$ is contained in a connected component of $|K| \cap S$, which in turn is contained in some simplex $\Delta \in K$ by construction of $\mathscr{S}_{K}$.

We are now ready to prove the first two theorems announced in Sect. 1. 
Proof of Theorem 1.3 Let $n$ be a positive integer. We may assume that $V \subseteq \mathbb{R}^{m}$ and $\mathbb{G}\left(\mathbb{F}^{n}\right) \subseteq \mathbb{R}^{N}$ are Zariski closed subvarieties.

Consider a continuous map $f: X \rightarrow \mathbb{G}\left(\mathbb{F}^{n}\right) \subseteq \mathbb{R}^{N}$. By Tietze's extension theorem, there exists a continuous map $F: \mathbb{R}^{m} \rightarrow \mathbb{R}^{N}$ with $\left.F\right|_{X}=f$. Let $\rho: T \rightarrow \mathbb{G}\left(\mathbb{F}^{n}\right)$ be a tubular neighborhood of $\mathbb{G}\left(\mathbb{F}^{n}\right)$ in $\mathbb{R}^{N}$. Then $U:=F^{-1}(T) \subseteq \mathbb{R}^{m}$ is a neighborhood of $X$, and $\tilde{f}: U \rightarrow \mathbb{G}\left(\mathbb{F}^{n}\right)$, given by $\tilde{f}(x)=\rho(F(x))$ for $x \in U$, is a continuous extension of $f$. Since $X$ is a compact subset of $U$, we get $X \subseteq B \subseteq U$, where $B$ is the union of a finite collection of simplices in $\mathbb{R}^{m}$. Then there exists a finite simplicial complex $K$ in $\mathbb{R}^{m}$ with $|K|=B$. In view of Proposition 4.3, there exists a continuous map $\varphi:|K| \rightarrow \mathbb{G}\left(\mathbb{F}^{n}\right)$, arbitrarily close to $\left.\tilde{f}\right|_{|K|}$, such that the restriction $\left.\varphi\right|_{\Delta}: \Delta \rightarrow \mathbb{G}\left(\mathbb{F}^{n}\right)$ is a regular map for every simplex $\Delta \in K$. According to Lemma 4.4, the restriction $g:=\left.\varphi\right|_{X}: X \rightarrow \mathbb{G}\left(\mathbb{F}^{n}\right)$ is a piecewise $\mathscr{S}_{K}(V)$-regular map. The proof is complete since $g$ is close to $f$.

Proof of Theorem 1.4 It can be assumed that $V \subseteq \mathbb{R}^{m}$ is a Zariski closed subvariety, hence $X \subseteq \mathbb{R}^{m}$. By Borsuk's theorem [26, p. 537], $X$ is a retract of some neighborhood $U \subseteq \mathbb{R}^{m}$. We can find a finite simplicial complex $K$ in $\mathbb{R}^{m}$ with $X \subseteq|K| \subseteq U$. Thus there exists a retraction $r:|K| \rightarrow X$.

We claim that the induced stratification $\mathscr{S}:=\mathscr{S}_{K}(V)$ of $V$ has all the required properties. Indeed, let $n$ be a positive integer and let $f: X \rightarrow \mathbb{G}\left(\mathbb{F}^{n}\right)$ be a continuous map. Then $f \circ r:|K| \rightarrow \mathbb{G}\left(\mathbb{F}^{n}\right)$ is a continuous extension of $f$. By Proposition 4.3, there exists a continuous map $\varphi:|K| \rightarrow \mathbb{G}\left(\mathbb{F}^{n}\right)$, arbitrarily close to $f \circ r$, such that the restriction $\left.\varphi\right|_{\Delta}: \Delta \rightarrow \mathbb{G}\left(\mathbb{F}^{n}\right)$ is a regular map for every simplex $\Delta \in K$. In view of Lemma 4.4, the restriction $g:=\left.\varphi\right|_{X}: X \rightarrow \mathbb{G}\left(\mathbb{F}^{n}\right)$ is a piecewise $\mathscr{S}$-regular map. This completes the proof since $g$ is close to $f$.

We also have the following variant of Theorem 1.4.

Theorem 4.5 Let $X_{0} \subseteq \mathbb{R}^{m}$ be a compact subset, $U \subseteq \mathbb{R}^{m}$ a neighborhood of $X_{0}$, $\beta: U \rightarrow U$ a homeomorphism, and $K$ is a finite simplicial complex in $\mathbb{R}^{m}$. Assume that $X_{0}$ is a retract of $U$ and

$$
X:=\beta\left(X_{0}\right) \subseteq|K| \subseteq U
$$

Then, for each positive integer $n$, every continuous map from $X$ into $\mathbb{G}\left(\mathbb{F}^{n}\right)$ can be approximated by piecewise $\mathscr{S}_{K}$-regular maps.

Proof Let $r_{0}: U \rightarrow X_{0}$ be a retraction. Then $r_{X}: U \rightarrow X$, given by

$$
r_{X}(x)=\beta\left(r_{0}\left(\beta^{-1}(x)\right)\right) \text { for all } x \in U,
$$

is a well-defined retraction.

Let $n$ be a positive integer and let $f: X \rightarrow \mathbb{G}\left(\mathbb{F}^{n}\right)$ be a continuous map. Since $f \circ r_{X}: U \rightarrow \mathbb{G}\left(\mathbb{F}^{n}\right)$ is a continuous extension of $f$, we complete the proof as in the case of Theorem 1.4.

Theorem 4.5 can be illustrated by revisiting Example 1.7. 
Example 4.6 Fix an integer $m \geq d(\mathbb{F})+1$, and consider $\mathbb{T}^{m}$ as a subset of $\mathbb{R}^{2 m} \times \mathbb{R}=$ $\mathbb{R}^{2 m+1}$. Note that $\mathbb{T}^{m}$ is a retract of $U:=(\mathbb{R} \backslash\{0\})^{2 m} \times \mathbb{R}$. Let $K$ be a finite simplicial complex in $\mathbb{R}^{2 m+1}$ satisfying

$$
\mathbb{T}^{m} \subseteq \operatorname{int}|K| \subseteq|K| \subseteq U
$$

According to Example 1.7, we can find a nonsingular Zariski closed subvariety $V \subseteq$ $\mathbb{R}^{2 m+1}$, a $\mathscr{C}^{\infty}$ diffeomorphism $\beta: U \rightarrow U$, and a $\mathscr{C}^{\infty}$ map

$$
h: V \rightarrow \mathbb{G}_{1}\left(\mathbb{F}^{2}\right)=\mathbb{S}^{d(\mathbb{F})}
$$

such that $V=\beta\left(\mathbb{T}^{m}\right) \subseteq|K|$ and $h$ is not homotopic to any stratified-regular map.

By Theorem 4.5, for each positive integer $n$, every continuous map from $V$ into $\mathbb{G}\left(\mathbb{F}^{n}\right)$ can be approximated by piecewise $\mathscr{S}_{K}$-regular maps. In particular, this is the case for the map $h$.

\section{Piecewise-algebraic vector bundles}

Comparison of algebraic and topological vector bundles on a given real algebraic variety $V$ is a challenging problem, cf. [2-6,11,12, 14,21,22, 25, 27,32,42,47-49, 77, $79,80]$. Unless the variety $V$ is quite exceptional, one can find a topological vector bundle on $V$ that is not topologically isomorphic to any algebraic vector bundle. On the other hand, in some sense, algebraization of topological vector bundles is possible, cf. $[2,49,69,70,79,80]$. In this section we look at the algebraization problem from a new perspective. The main results are Theorems 5.10 and 5.11, derived from Theorems 1.3 and 1.4 , respectively.

We will use freely notation introduced in Sect. 4. Moreover, given a topological space $X$, a subspace $A \subseteq X$, and a topological morphism $\psi: \theta \rightarrow \omega$ of topological $\mathbb{F}$ vector bundles on $X$, we let $\psi_{A}:\left.\left.\theta\right|_{A} \rightarrow \omega\right|_{A}$ denote the restriction morphism defined by $\psi_{A}(v)=\psi(v)$ for all $v \in E\left(\left.\theta\right|_{A}\right)$.

We first generalize the definition of algebraic vector bundle.

Definition 5.1 Let $V$ be a real algebraic variety, $X \subseteq V$ some (nonempty) subset, and $Z$ the Zariski closure of $X$ in $V$.

An algebraic $\mathbb{F}$-vector bundle $\xi$ on $X$ is a topological $\mathbb{F}$-vector subbundle of $\varepsilon_{X}^{n}(\mathbb{F})$, for some $n$, for which there exist a Zariski open neighborhood $Z_{0} \subseteq Z$ of $X$ and an algebraic $\mathbb{F}$-vector subbundle $\tilde{\xi}$ of $\varepsilon_{Z_{0}}^{n}(\mathbb{F})$ with $\left.\tilde{\xi}\right|_{X}=\xi$. In that case, $\xi$ is also said to be an algebraic $\mathbb{F}$-vector subbundle of $\varepsilon_{X}^{n}(\mathbb{F})$. The pair $\left(Z_{0}, \tilde{\xi}\right)$ is called an algebraic extension of $\xi$. In particular, $\varepsilon_{X}^{n}(\mathbb{F})$ is an algebraic $\mathbb{F}$-vector bundle on $X$.

If $\xi$ and $\eta$ are algebraic $\mathbb{F}$-vector bundles on $X$, then an algebraic morphism $\varphi: \xi \rightarrow$ $\eta$ is a topological morphism such that there exist algebraic extensions $\left(Z_{0}, \tilde{\xi}\right),\left(Z_{0}, \tilde{\eta}\right)$ of $\xi, \eta$, respectively, and an algebraic morphism $\tilde{\varphi}: \tilde{\xi} \rightarrow \tilde{\eta}$ with $\tilde{\varphi}_{X}=\varphi$.

Algebraic $\mathbb{F}$-vector bundles on $X$, together with algebraic morphisms, form a category. 
Lemma 5.2 Let $V$ be a real algebraic variety, $X \subseteq V$ some subset, and $\varphi: \xi \rightarrow \eta$ a bijective algebraic morphism of algebraic $\mathbb{F}$-vector bundles on $X$. Then $\varphi$ is an algebraic isomorphism.

Proof Let $\left(Z_{0}, \tilde{\xi}\right),\left(Z_{0}, \tilde{\eta}\right)$, and $\tilde{\varphi}: \tilde{\xi} \rightarrow \tilde{\eta}$ be as in Definition 5.1. Shrinking $Z_{0}$ if necessary, we may assume that the algebraic morphism $\tilde{\varphi}$ is bijective. Thus the proof is reduced to the case where $X$ is a Zariski locally closed subvariety of $V$.

Our goal is to prove that the inverse map $\varphi^{-1}: E(\eta) \rightarrow E(\xi)$ is a regular map. The problem is local for the Zariski topology on $X$, so it suffices to consider $\xi=\eta=\varepsilon_{X}^{n}(\mathbb{F})$. Let $A=A_{\varphi}: X \rightarrow \operatorname{Mat}_{n}(\mathbb{F})$ be the matrix representation of $\varphi$. Then $A$ is a regular map and

$$
\varphi^{-1}(x, w)=\left(x, A(x)^{-1}(w)\right) \text { for }(x, w) \in X \times \mathbb{F}^{n} .
$$

Consequently, $\varphi^{-1}$ is a regular map, as required.

Lemma 5.3 Let $V$ be a real algebraic variety, $X \subseteq V$ some subset, and $\xi$ an algebraic $\mathbb{F}$-vector subbundle of $\varepsilon_{X}^{n}(\mathbb{F})$. Then the orthogonal complement $\xi^{\perp}$ of $\xi$ is also an algebraic $\mathbb{F}$-vector subbundle of $\varepsilon_{X}^{n}(\mathbb{F})$, and the orthogonal projection $\rho_{\xi}: \varepsilon_{X}^{n}(\mathbb{F}) \rightarrow$ $\varepsilon_{X}^{n}(\mathbb{F})$ onto $\xi$ is an algebraic morphism.

Proof This is a standard fact if $X$ is a Zariski locally closed subvariety of $V$. The general case follows immediately.

We denote by $\gamma\left(\mathbb{F}^{n}\right)$ the algebraic $\mathbb{F}$-vector subbundle of $\varepsilon_{\left.\mathbb{G}^{(} \mathbb{F}^{n}\right)}^{n}(\mathbb{F})$ whose restriction to $\mathbb{G}_{r}\left(\mathbb{F}^{n}\right)$ is $\gamma_{r}\left(\mathbb{F}^{n}\right)$ for $0 \leq r \leq n$.

Given a topological space $X$ and a continuous map $f: X \rightarrow \mathbb{G}\left(\mathbb{F}^{n}\right)$, we regard the pullback $f^{*} \gamma\left(\mathbb{F}^{n}\right)$ as a topological $\mathbb{F}$-vector subbundle of $\varepsilon_{X}^{n}(\mathbb{F})$; thus

$$
E\left(f^{*} \gamma\left(\mathbb{F}^{n}\right)\right)_{x}=\{x\} \times f(x) \text { for all } x \in X \text {. }
$$

Conversely, if $\xi$ is a topological $\mathbb{F}$-vector subbundle of $\varepsilon_{X}^{n}(\mathbb{F})$, then the map $f_{\xi}: X \rightarrow$ $\mathbb{G}\left(\mathbb{F}^{n}\right)$, defined by

$$
E(\xi)_{x}=\{x\} \times f_{\xi}(x) \text { for all } x \in X
$$

is continuous and $\xi=f_{\xi}^{*} \gamma\left(\mathbb{F}^{n}\right)$. We call $f_{\xi}$ the classifying map for $\xi$. Note that the classifying map for $f^{*} \gamma\left(\mathbb{F}^{n}\right)$ is $f$.

Lemma 5.4 Let $V$ be a real algebraic variety, $X \subseteq V$ some subset, and $\xi$ a topological $\mathbb{F}$-vector subbundle of $\varepsilon_{X}^{n}(\mathbb{F})$. Then the following conditions are equivalent:

(a) $\xi$ is an algebraic $\mathbb{F}$-vector subbundle of $\varepsilon_{X}^{n}(\mathbb{F})$.

(b) The classifying map $f_{\xi}: X \rightarrow \mathbb{G}\left(\mathbb{F}^{n}\right)$ for $\xi$ is regular.

Proof This is well-known if $X$ is a Zariski locally closed subvariety of $V$. The general case follows immediately.

Next we introduce the central notion of this section. 
Definition 5.5 Let $V$ be a real algebraic variety, $X \subseteq V$ some subset, and $\mathscr{S}$ a stratification of $V$.

An $\mathscr{S}$-algebraic (resp. a piecewise $\mathscr{S}$-algebraic) $\mathbb{F}$-vector bundle $\xi$ on $X$ is a topological $\mathbb{F}$-vector subbundle of $\varepsilon_{X}^{n}(\mathbb{F})$, for some $n$, such that for every stratum $S \in \mathscr{S}$ the restriction $\left.\xi\right|_{X \cap S}$ is an algebraic $\mathbb{F}$-vector subbundle of $\varepsilon_{X \cap S}^{n}(\mathbb{F})$ (resp. for every stratum $S \in \mathscr{S}$ and each connected component $\Sigma$ of $X \cap S$ the restriction $\left.\xi\right|_{\Sigma}$ is an algebraic $\mathbb{F}$-vector subbundle of $\varepsilon_{\Sigma}^{n}(\mathbb{F})$ ). In that case, $\xi$ is also said to be an $\mathscr{S}$-algebraic (resp. a piecewise $\mathscr{S}$-algebraic) $\mathbb{F}$-vector subbundle of $\varepsilon_{X}^{n}(\mathbb{F})$.

If $\xi$ and $\eta$ are $\mathscr{S}$-algebraic (resp. piecewise $\mathscr{S}$-algebraic) $\mathbb{F}$-vector bundles on $X$, then an $\mathscr{S}$-algebraic (resp. a piecewise $\mathscr{S}$-algebraic) morphism $\varphi: \xi \rightarrow \eta$ is a topological morphism such that for every stratum $S \in \mathscr{S}$ the restriction $\varphi_{X \cap S}:\left.\left.\xi\right|_{X \cap S} \rightarrow \eta\right|_{X \cap S}$ is an algebraic morphism (resp. for every stratum $S \in \mathscr{S}$ and each connected component $\Sigma$ of $X \cap S$ the restriction $\varphi_{\Sigma}:\left.\left.\xi\right|_{\Sigma} \rightarrow \eta\right|_{\Sigma}$ is an algebraic morphism).

A stratified-algebraic (resp. a piecewise-algebraic) $\mathbb{F}$-vector bundle on $X$ is a $\mathscr{T}$ algebraic (resp. a piecewise $\mathscr{T}$-algebraic) $\mathbb{F}$-vector bundle on $X$ for some stratification $\mathscr{T}$ of $V$.

If $\xi$ and $\eta$ are stratified-algebraic (resp. piecewise-algebraic) $\mathbb{F}$-vector bundles on $X$, then a stratified-algebraic (resp. a piecewise-algebraic) morphism $\varphi: \xi \rightarrow \eta$ is a $\mathscr{T}$-algebraic (resp. a piecewise $\mathscr{T}$-algebraic) morphism for some stratification $\mathscr{T}$ of $V$ such that both $\xi$ and $\eta$ are $\mathscr{T}$-algebraic (resp. piecewise $\mathscr{T}$-algebraic) $\mathbb{F}$-vector bundles on $X$.

It is clear that $\mathscr{S}$-algebraic (resp. piecewise $\mathscr{S}$-algebraic) $\mathbb{F}$-vector bundles on $X$, together with $\mathscr{S}$-algebraic (resp. piecewise $\mathscr{S}$-algebraic) morphisms, form a category. Similarly, stratified-algebraic (resp. piecewise-algebraic) $\mathbb{F}$-vector bundles on $X$, together with stratified-algebraic (resp. piecewise-algebraic) morphisms, form a category.

In a somewhat less general context, $\mathscr{S}$-algebraic and stratified-algebraic $\mathbb{F}$-vector bundles are thoroughly investigated in $[57,59,61,63,66]$.

Proposition 5.6 Let $V$ be a real algebraic variety, $X \subseteq V$ some subset, $\mathscr{S}$ a stratification of $V$, and $\xi$ a topological $\mathbb{F}$-vector subbundle of $\varepsilon_{X}^{n}(\mathbb{F})$ for some $n$. Then the following conditions are equivalent:

(a) $\xi$ is an $\mathscr{S}$-algebraic (resp. a piecewise $\mathscr{S}$-algebraic) $\mathbb{F}$-vector subbundle of $\varepsilon_{X}^{n}(\mathbb{F})$.

(b) The classifying map $f_{\xi}: X \rightarrow \mathbb{G}\left(\mathbb{F}^{n}\right)$ for $\xi$ is $\mathscr{S}$-regular (resp. piecewise $\mathscr{S}$ regular).

Proof This follows from Lemma 5.4.

As a direct consequence, we obtain the following.

Corollary 5.7 Let $V$ be a real algebraic variety, $X \subseteq V$ some subset, and $\xi$ a topological $\mathbb{F}$-vector subbundle of $\varepsilon_{X}^{n}(\mathbb{F})$ for some $n$. Then the following conditions are 
equivalent:

(a) $\xi$ is a stratified-algebraic (resp. a piecewise-algebraic) $\mathbb{F}$-vector subbundle of $\varepsilon_{X}^{n}(\mathbb{F})$.

(b) The classifying map $f_{\xi}: X \rightarrow \mathbb{G}\left(\mathbb{F}^{n}\right)$ for $\xi$ is stratified-regular (resp. piecewiseregular).

Vector bundles introduced in Definition 5.5 can be compared with topological vector bundles.

Proposition 5.8 Let $V$ be a real algebraic variety, $X \subseteq V$ a compact subset, and $\mathscr{S} a$ stratification of $V$. Let $\xi$ and $\eta$ be $\mathscr{S}$-algebraic (resp. piecewise $\mathscr{S}$-algebraic) $\mathbb{F}$-vector bundles on $X$ that are topologically isomorphic. Then $\xi$ and $\eta$ are also isomorphic in the category of $\mathscr{S}$-algebraic (resp. piecewise $\mathscr{S}$-algebraic) $\mathbb{F}$-vector bundles on $X$.

Proof We consider explicitly only the piecewise $\mathscr{S}$-algebraic case, the $\mathscr{S}$-algebraic one being completely analogous.

Thus, $\xi$ (resp. $\eta$ ) is a piecewise $\mathscr{S}$-algebraic $\mathbb{F}$-vector subbundle of $\varepsilon_{X}^{k}(\mathbb{F})$ (resp. $\left.\varepsilon_{X}^{l}(\mathbb{F})\right)$ for some positive integer $k\left(\right.$ resp. $l$ ). Since $\varepsilon_{X}^{k}(\mathbb{F})=\xi \oplus \xi^{\perp}$ and $\varepsilon_{X}^{l}(\mathbb{F})=\eta \oplus \eta^{\perp}$, there exists a topological morphism $\varphi: \varepsilon_{X}^{k}(\mathbb{F}) \rightarrow \varepsilon_{X}^{l}(\mathbb{F})$ which transforms $\xi$ onto $\eta$. Let $A=A_{\varphi}: X \rightarrow \operatorname{Mat}_{l, k}(\mathbb{F})$ be the matrix representation of $\varphi$. By the Weierstrass approximation theorem, we can find a regular map $B: X \rightarrow \operatorname{Mat}_{l, k}(\mathbb{F})$ that is close to $A$. Then $\psi: \varepsilon_{X}^{k}(\mathbb{F}) \rightarrow \varepsilon_{X}^{l}(\mathbb{F})$, defined by

$$
\psi(x, v)=(x, B(x)(v)) \text { for }(x, v) \in X \times \mathbb{F}^{k},
$$

is an algebraic morphism.

In view of Lemma 5.3, the orthogonal projection $\rho_{\eta}: \varepsilon_{X}^{l}(\mathbb{F}) \rightarrow \varepsilon_{X}^{l}(\mathbb{F})$ onto $\eta$ is a piecewise $\mathscr{S}$-algebraic morphism. Hence $\rho_{\eta} \circ \psi: \varepsilon_{X}^{k}(\mathbb{F}) \rightarrow \varepsilon_{X}^{l}(\mathbb{F})$ is a piecewise $\mathscr{S}$-algebraic morphism which transforms $\xi$ onto $\eta$. Consequently, the morphism $\sigma: \xi \rightarrow \eta$ determined by $\rho_{\eta} \circ \psi$ is bijective and piecewise $\mathscr{S}$-algebraic. It follows from Lemma 5.2 that $\sigma$ is a piecewise $\mathscr{S}$-algebraic isomorphism.

Proposition 5.8 implies immediately the following.

Corollary 5.9 Let $V$ be a real algebraic variety, and $X \subseteq V$ a compact subset. Let $\xi$ and $\eta$ be stratified-algebraic (resp. piecewise-algebraic) $\mathbb{F}$-vector bundles on $X$ that are topologically isomorphic. Then $\xi$ and $\eta$ are also isomorphic in the category of stratified-algebraic (resp. piecewise-algebraic) $\mathbb{F}$-vector bundles on X.

As an application of Theorem 1.3, we obtain the following result.

Theorem 5.10 Let $V$ be a real algebraic variety and let $X \subseteq V$ be a compact subset. Then each topological $\mathbb{F}$-vector bundle on $X$ is topologically isomorphic to a piecewise-algebraic $\mathbb{F}$-vector bundle on $X$, which is uniquely determined up to piecewise-algebraic isomorphism. 
Proof Let $\xi$ be a topological $\mathbb{F}$-vector bundle on $X$. Since $X$ is compact, one can find a positive integer $n$ and a continuous map $f: X \rightarrow \mathbb{G}\left(\mathbb{F}^{n}\right)$ such that $\xi$ is topologically isomorphic to the pullback $f^{*} \gamma\left(\mathbb{F}^{n}\right)$, cf. [39, Chapter 3, Proposition 5.8]. According to Theorem 1.3, $f$ is homotopic to a piecewise-regular map $g: X \rightarrow \mathbb{G}\left(\mathbb{F}^{n}\right)$, hence $\xi$ is topologically isomorphic to the pullback $\eta:=g^{*} \gamma\left(\mathbb{F}^{n}\right)$, cf. [39, Chapter 3, Theorem 4.7]. By Corollary 5.7, $\eta$ is a piecewise-algebraic $\mathbb{F}$-vector bundle on $X$. The proof is complete in view of Corollary 5.9.

In a similar way, we can derive from Theorem 1.4 the next result.

Theorem 5.11 Let $V$ be a real algebraic variety and let $X \subseteq V$ be a compact locally contractible subset. Then there exists a stratification $\mathscr{S}$ of $V$ such that each topological $\mathbb{F}$-vector bundle on $X$ is topologically isomorphic to a piecewise $\mathscr{S}$-algebraic $\mathbb{F}$-vector bundle on $X$, which is uniquely determined up to piecewise $\mathscr{S}$-algebraic isomorphism.

Proof According to Theorem 1.4, there exists a stratification $\mathscr{S}$ of $V$ such that, for each positive integer $n$, every continuous map from $X$ into $\mathbb{G}\left(\mathbb{F}^{n}\right)$ is homotopic to a piecewise $\mathscr{S}$-regular map.

Let $\xi$ be a topological $\mathbb{F}$-vector bundle on $X$. One can find a positive integer $n$ and a continuous map $f: X \rightarrow \mathbb{G}\left(\mathbb{F}^{n}\right)$ such that $\xi$ is topologically isomorphic to the pullback $f^{*} \gamma\left(\mathbb{F}^{n}\right)$. Hence $\xi$ is topologically isomorphic to the pullback $\eta:=g^{*} \gamma\left(\mathbb{F}^{n}\right)$, where $g: X \rightarrow \mathbb{G}\left(\mathbb{F}^{n}\right)$ is a piecewise $\mathscr{S}$-regular map homotopic to $f$. By Proposition 5.6, $\eta$ is a piecewise $\mathscr{S}$-algebraic $\mathbb{F}$-vector bundle on $X$. The proof is complete in view of Proposition 5.8.

In Theorems 5.10 and 5.11, piecewise-algebraic and piecewise $\mathscr{S}$-algebraic cannot be replaced by stratified-algebraic and $\mathscr{S}$-algebraic, respectively.

Example 5.12 Fix an integer $m \geq d(\mathbb{F})+1$, and let $V$ and $h: V \rightarrow \mathbb{G}_{1}\left(\mathbb{F}^{2}\right)=\mathbb{S}^{d}(\mathbb{F})$ be as in Example 1.7. We claim that the topological $\mathbb{F}$-vector bundle $\xi:=h^{*} \gamma_{1}\left(\mathbb{F}^{2}\right)$ on $V$ is not topologically isomorphic to any stratified-algebraic $\mathbb{F}$-vector bundle. Indeed, write $\xi_{\mathbb{R}}$ and $\gamma_{1}\left(\mathbb{F}^{2}\right)_{\mathbb{R}}$ to indicate that $\xi$ and $\gamma_{1}\left(\mathbb{F}^{2}\right)$ are regarded as topological $\mathbb{R}$-vector bundles. Since $w_{d(\mathbb{F})}\left(\gamma_{1}\left(\mathbb{F}^{2}\right)_{\mathbb{R}}\right)=s_{d(\mathbb{F})}$, we get

$$
w_{d(\mathbb{F})}\left(\xi_{\mathbb{R}}\right)=h^{*}\left(s_{d(\mathbb{F})}\right) \notin H_{\mathrm{alg}}^{d(\mathbb{F})}(V ; \mathbb{Z} / 2),
$$

where $w_{q}(-)$ stands for the $q$ th Stiefel-Whitney class. The claim follows in view of [61, Propositions 7.3 and 7.7].

\section{Piecewise-regular maps into spheres (approximation)}

For maps with values in $\mathbb{S}^{n}$, we have the following counterpart of Proposition 4.3.

Proposition 6.1 Let $K$ be a finite simplicial complex in $\mathbb{R}^{m}$ and let $f:|K| \rightarrow \mathbb{S}^{n}$ be a continuous map. Assume that $f(\Delta) \neq \mathbb{S}^{n}$ for every simplex $\Delta \in K$. Then, for each open neighborhood $\mathscr{U} \subseteq \mathscr{C}\left(|K|, \mathbb{S}^{n}\right)$ of $f$, there exists a continuous map $\varphi:|K| \rightarrow \mathbb{S}^{n}$ such that $\varphi \in \mathscr{U}$ and the restriction $\left.\varphi\right|_{\Delta}: \Delta \rightarrow \mathbb{S}^{n}$ is a regular map for every simplex $\Delta \in K$. 
Proof We use induction on $d=\operatorname{dim} K$. The case $d=0$ is obvious. Suppose now that $d \geq 1$. By the induction hypothesis, there exists a continuous map $\psi:\left|K^{(d-1)}\right| \rightarrow \mathbb{S}^{n}$, arbitrarily close to $\left.f\right|_{\left|K^{(d-1)}\right|}$, such that the restriction $\left.\psi\right|_{\Gamma}: \Gamma \rightarrow \mathbb{S}^{n}$ is a regular map for every simplex $\Gamma \in K^{(d-1)}$. It readily follows that $\psi$ can be extended to a continuous map $\tilde{\psi}:|K| \rightarrow \mathbb{S}^{n}$ that belongs to $\mathscr{U}$ and satisfies $\tilde{\psi}(\Delta) \neq \mathbb{S}^{n}$ for every simplex $\Delta \in K$. Since $\mathbb{S}^{n}$ with one point removed is biregularly isomorphic to $\mathbb{R}^{n}$, it follows from Lemma 3.7 that for every $d$-simplex $\Delta \in K$, there exists a regular map $g_{\Delta}: \Delta \rightarrow \mathbb{S}^{n}$ close to $\left.\tilde{\psi}\right|_{\Delta}$ and such that $\left.g_{\Delta}\right|_{|\dot{\Delta}|}=\left.\tilde{\psi}\right|_{|\dot{\Delta}|}$. Then the map $\varphi:|K| \rightarrow \mathbb{S}^{n}$, defined by $\left.\varphi\right|_{\left|K^{(d-1)}\right|}=\psi$ and $\left.\varphi\right|_{\Delta}=g_{\Delta}$ for every $d$-simplex $\Delta \in K$, has all the required properties.

In what follows we will make use of Lemma 4.4.

Proof of Theorem 1.5 Let $n$ be a positive integer and let $f: X \rightarrow \mathbb{S}^{n} \subseteq \mathbb{R}^{n+1}$ be a continuous map. We may assume that $V \subseteq \mathbb{R}^{m}$ is a Zariski closed subvariety, hence $X \subseteq \mathbb{R}^{m}$. By Tietze's extension theorem, there exists a continuous map $F: \mathbb{R}^{m} \rightarrow$ $\mathbb{R}^{n+1}$ with $\left.F\right|_{X}=f$. Denoting by $\rho: \mathbb{R}^{n+1} \backslash\{0\} \rightarrow \mathbb{S}^{n}$ the radial projection, we see that $U:=F^{-1}\left(\mathbb{R}^{n+1} \backslash\{0\}\right) \subseteq \mathbb{R}^{m}$ is a neighborhood of $X$, and $\tilde{f}: U \rightarrow \mathbb{S}^{n}$, given by $\tilde{f}(x)=\rho(F(x))$ for $x \in U$, is a continuous extension of $f$.

We can find a finite simplicial complex $K$ in $\mathbb{R}^{m}$ with $X \subseteq|K| \subseteq U$. By replacing $K$ with a suitable iterated barycentric subdivision of $K$, we get $\tilde{f}(\Delta) \neq \mathbb{S}^{n}$ for every simplex $\Delta \in K$. In view of Proposition 6.1, we can find a continuous map $\varphi:|K| \rightarrow \mathbb{S}^{n}$, arbitrarily close to $\left.\tilde{f}\right|_{|K|}$, such that the restriction $\left.\varphi\right|_{\Delta}: \Delta \rightarrow \mathbb{S}^{n}$ is a regular map for every simplex $\Delta \in K$. According to Lemma 4.4, the restriction $g:=\left.\varphi\right|_{X}: X \rightarrow \mathbb{S}^{n}$ is a piecewise $\mathscr{S}_{K}(V)$-regular map. The proof is complete since $g$ is close to $f$.

It is not clear whether there is a counterpart of Theorem 1.4 for maps into spheres.

Problem 6.2 Let $V$ be a real algebraic variety, $X \subseteq V$ a compact locally contractible subset, and $n$ a positive integer. Does there exist a stratification $\mathscr{S}$ of $V$ such that every continuous map from $X$ into $\mathbb{S}^{n}$ can be approximated by piecewise $\mathscr{S}$-regular maps?

In view of Theorem 1.4, the answer is affirmative if $n=1,2$ or 4 since $\mathbb{S}^{d(\mathbb{F})}=$ $\mathbb{G}_{1}\left(\mathbb{F}^{2}\right)$.

\section{Piecewise-regular maps into spheres (homotopy)}

The following lemma will be used in the proof of Theorem 1.6.

Lemma 7.1 Let $V$ be a compact nonsingular real algebraic variety, $Z \subseteq V$ a nonsingular Zariski closed subvariety with $\operatorname{codim}_{V} Z \geq 1$, and $K$ the union of some connected components of $Z$. Then there exists a closed tubular neighborhood $T \subseteq V$ of $K$ such that $T \cap(Z \backslash K)=\varnothing$ and the boundary $\partial T$ of $T$ is a nonsingular Zariski closed subvariety of $V$. Furthermore, for each nonnegative integer $l$, there exists a $\mathscr{C}^{l}$ function $\alpha: V \rightarrow \mathbb{R}$ with the following properties:

(i) $Z(\alpha)=K$; 
(ii) the restrictions $\left.\alpha\right|_{T}$ and $\left.\alpha\right|_{V \backslash \text { intT }}$ are regular functions.

Proof Let $T \subseteq V$ be a closed $\mathscr{C}^{\infty}$ tubular neighborhood of $K$ with $T \cap(Z \backslash K)=\varnothing$. Note that the homology class in $H_{*}(V ; \mathbb{Z} / 2)$ represented by the $\mathscr{C}^{\infty}$ hypersurface $\partial T \subseteq V$ is equal to 0 . Hence there exists a $\mathscr{C}^{\infty}$ diffeomorphism $\sigma: V \rightarrow V$, arbitrarily close in the $\mathscr{C}^{\infty}$ topology to the identity map of $V$, such that $\sigma(\partial T)$ is a nonsingular Zariski closed subvariety of $V$ and $\sigma(x)=x$ for all $x \in Z$, cf. [6, Theorem 12.4.11]. Replacing $T$ by $\sigma(T)$, we may assume that $\partial T$ is a nonsingular Zariski closed subvariety of $V$.

Let $f$ and $g$ be real-valued regular functions on $V$ with $Z(f)=Z$ and $Z(g)=\partial T$. If $l$ is a nonnegative integer, then

$$
\alpha(x)= \begin{cases}f(x)^{2} & \text { for } x \in T \\ f(x)^{2}+g(x)^{2 l} & \text { for } x \in V \backslash T\end{cases}
$$

is a $\mathscr{C}^{l}$ function that satisfies (i) and (ii).

Our proof of Theorem 1.6 depends on the Pontryagin-Thom construction. Unless explicitly stated otherwise, all $\mathscr{C}^{\infty}$ manifolds will be without boundary. Submanifolds will be closed subsets of the ambient manifold. The unit $n$-sphere $\mathbb{S}^{n}$ will be oriented as the boundary of the unit $(n+1)$-disc. For any compact $\mathscr{C}^{\infty}$ manifold $X$ there is a canonical one-to-one correspondence

$$
\pi^{n}(X) \rightarrow F^{n}(X)
$$

where $\pi^{n}(X)$ is the set of all homotopy classes of continuous maps from $X$ into $\mathbb{S}^{n}$, and $F^{n}(X)$ is the set of framed cobordism classes of framed submanifolds of $X$ of codimension $n$, cf. $[46,73]$ for details. Given a continuous map $f: X \rightarrow \mathbb{S}^{n}$, we denote by PT $(f)$ the element of $F^{n}(X)$ corresponding to the homotopy class of $f$.

It is convenient to introduce some notation related to this construction. A framed submanifold of $X$ of codimension $n$ is a pair $(M, F)$, where $M \subseteq X$ is a $\mathscr{C}^{\infty}$ codimension $n$ submanifold, and $F=\left(v_{1}, \ldots, v_{n}\right)$ is a $\mathscr{C}^{\infty}$ framing of the normal bundle to $M$ in $X$. The normal space to $M$ in $X$ at $x \in M$ is the quotient $N_{x} M:=T_{x} X / T_{x} M$ of the tangent spaces; thus $\left(v_{1}(x), \ldots, v_{n}(x)\right)$ is a basis for $N_{x} M$.

Given a continuous map $f: X \rightarrow \mathbb{S}^{n}$ and a point $y \in \mathbb{S}^{n}$, suppose that for some open neighborhood $U \subseteq \mathbb{S}^{n}$ of $y$ the restriction map $\left.f\right|_{f^{-1}(U)}: f^{-1}(U) \rightarrow$ $U$ is of class $\mathscr{C}^{\infty}$ and transverse to $y$. Choose a positively oriented basis $B=\left(w_{1}, \ldots, w_{n}\right)$ for $T_{y} \mathbb{S}^{n}$. Then $\mathrm{PT}(f) \in F^{n}(X)$ is represented by the framed submanifold $\left(f^{-1}(y), F(f, B)\right)$, where $F(f, B)=\left(v_{1}, \ldots, v_{n}\right)$ and $\left(v_{1}(x), \ldots, v_{n}(x)\right)$ is transformed onto $\left(w_{1}, \ldots, w_{n}\right)$ by the isomorphism $N_{x} M \rightarrow T_{y} \mathbb{S}^{n}$ induced by the derivative $d_{x} f: T_{x} X \rightarrow T_{y} \mathbb{S}^{n}$ for every $x \in f^{-1}(y)$.

Let $\varphi: X \rightarrow \mathbb{R}^{n}$ be a $\mathscr{C}^{\infty}$ map transverse to $0 \in \mathbb{R}^{n}$ and let $M$ be the union of some connected components of $\varphi^{-1}(0)$. Then we obtain a framed submanifold $(M, F(\varphi))$ of $X$, where $F(\varphi)=\left(v_{1}, \ldots, v_{n}\right)$ and $\left(v_{1}(x), \ldots, v_{n}(x)\right)$ is transformed onto the canonical basis for $\mathbb{R}^{n}$ by the isomorphism $N_{x} M \rightarrow \mathbb{R}^{n}$ induced by the derivative 
$d_{x} \varphi: T_{x} X \rightarrow T_{0} \mathbb{R}^{n}=\mathbb{R}^{n}$ for every $x \in M$. Note that the framing $F(\varphi)$ of $M$ can be defined even if the manifold $X$ is not necessarily compact.

Proof of Theorem 1.6 The case $\operatorname{dim} V<n$ is obvious since then $f$ is null homotopic. Suppose that $\operatorname{dim} V \geq n$, and let $(M, F)$ be a framed submanifold of $V$ which represents $\mathrm{PT}(f)$. Let $U \subseteq V$ be an open tubular neighborhood of $M$. Since $U$ is diffeomorphic to $M \times \mathbb{R}^{n}$, there exists a $\mathscr{C}^{\infty}$ map $\sigma: U \rightarrow \mathbb{R}^{n}$ transverse to $0 \in \mathbb{R}^{n}$ such that $M=\sigma^{-1}(0)$ and $(M, F)$ is framed cobordant to $(M, F(\sigma))$. By a standard transversality argument, we obtain a $\mathscr{C}^{\infty}$ map $\varphi: V \rightarrow \mathbb{R}^{n}$ which is transverse to $0 \in \mathbb{R}^{n}$ and $\varphi=\sigma$ on an open neighborhood of $M$ in $U$, cf. [36, Corollary 4.12 (b), p. 56]. Then $M$ is the union of some connected components of $\varphi^{-1}(0)$, and $(M, F)$ is framed cobordant to $(M, F(\varphi))=(M, F(\sigma))$. In view of the Weierstrass approximation theorem, there exists a regular map $\psi: V \rightarrow \mathbb{R}^{n}$ arbitrarily close to $\varphi$ in the $\mathscr{C}^{\infty}$ topology. Then $\psi$ is transverse to $0 \in \mathbb{R}^{n}$ and $Z:=\psi^{-1}(0)$ is isotopic to $\varphi^{-1}(0)$, cf. [1, p. 51]. In particular, $(M, F(\varphi))$ is framed cobordant to $(N, F(\psi))$, where $N$ is the union of suitable connected components of $Z$. Furthermore, $Z \subseteq V$ is a nonsingular Zariski closed subvariety. Set $K:=Z \backslash N$, and let $T, \alpha$ be as in Lemma 7.1 for some nonnegative integer $l$. According to the Łojasiewicz inequality [6, Corollary 2.6.7], we can find an open neighborhood $U \subseteq V$ of $K$, a real number $c>0$, and an integer $q>0$ such that

$$
\|\psi(x)\| \geq c \alpha(x)^{2 q} \text { for all } x \in U,
$$

where $\|-\|$ stands for the Euclidean norm on $\mathbb{R}^{n}$.

Set $a:=(0, \ldots, 0,1) \in \mathbb{S}^{n}, b:=-a$, and let $\rho: \mathbb{S}^{n} \backslash\{a\} \rightarrow \mathbb{R}^{n}$ be the stereographic projection. Fix a nonnegative integer $k$. If $l$ and $r$ are sufficiently large integers, then the map $g: V \rightarrow \mathbb{S}^{n}$ defined by

$$
g(x)= \begin{cases}\rho^{-1}\left(\frac{\psi(x)}{\alpha(x)^{2(q+r)}}\right) & \text { for } x \in V \backslash K \\ a & \text { for } x \in K\end{cases}
$$

is of class $\mathscr{C}^{k}$. Since $\rho$ is a biregular isomorphism, the restrictions $\left.g\right|_{T \backslash K}$ and $\left.g\right|_{V \backslash \operatorname{int} T}$ are regular maps. Consequently, $\mathscr{S}:=\{Z, \partial T, V \backslash(Z \cup \partial T)\}$ is a stratification of $V$, and the map $g$ is piecewise $\mathscr{S}$-regular.

It remains to prove that $g$ is homotopic to $f$ or, equivalently, $\mathrm{PT}(g)=\mathrm{PT}(f)$. To this end, let $B$ be the basis for $T_{b} \mathbb{S}^{n}$ which corresponds to the canonical basis for $\mathbb{R}^{n}$ via the isomorphism $d_{b} \rho: T_{b} \mathbb{S}^{n} \rightarrow \mathbb{R}^{n}$. Note that the restriction of $g$ to $g^{-1}\left(\mathbb{S}^{n} \backslash\{a\}\right)=V \backslash K$ is a $\mathscr{C}^{\infty}$ map transverse to $b \in \mathbb{S}^{n}$ and $g^{-1}(b)=N$. It readily follows that $(N, F(g, B))$ is framed cobordant to $(N, F(\varphi))$. Hence $(N, F(g, B))$ is framed cobordant to $(M, F)$, which implies that $\mathrm{PT}(g)=\mathrm{PT}(f)$, as required.

Remark 7.2 As demonstrated above, if $\operatorname{dim} V \geq n$, then the stratification $\mathscr{S}$ that appears in Theorem 1.6 can be chosen of the form

$$
\mathscr{S}=\{Z, W, V \backslash(Z \cup W)\}
$$

where $Z$ and $W$ are disjoint Zariski closed subvarieties of $V$ with $\operatorname{codim}_{V} Z=n$ and $\operatorname{codim}_{V} W=1$. 
Acknowledgements It is a pleasure to thank the referee for useful comments. The author was partially supported by the National Science Center (Poland) under Grant number 2014/15/ST1/00046.

Open Access This article is distributed under the terms of the Creative Commons Attribution 4.0 International License (http://creativecommons.org/licenses/by/4.0/), which permits unrestricted use, distribution, and reproduction in any medium, provided you give appropriate credit to the original author(s) and the source, provide a link to the Creative Commons license, and indicate if changes were made.

\section{References}

1. Abraham, R., Robbin, J.: Transversal Mappings and Flows. Benjamin, New York (1967)

2. Benedetti, R., Tognoli, A.: On real algebraic vector bundles. Bull. Sci. Math. 104(2), 89-112 (1980)

3. Benedetti, R., Tognoli, A.: Remarks and counterexamples in the theory of real vector bundles and cycles. In: Géométrie algébrique réelle et formes quadratiques, Lecture Notes in Math., vol. 959, pp. 198-211. Springer, Berlin (1982)

4. Bilski, M., Kucharz, W., Valette, A., Valette, G.: Vector bundles and regulous maps. Math. Z. 275, 403-418 (2013)

5. Bochnak, J., Buchner, M., Kucharz, W.: Vector bundles over real algebraic varieties. K-Theory 3, 271-298 (1989) [Erratum in K-Theory 4, 113 (1990)]

6. Bochnak, J., Coste, M., Roy, M.-F.: Real Algebraic Geometry, Ergeb. Math. Grenzgeb, vol. 36. Springer, Berlin (1989)

7. Bochnak, J., Kucharz, W.: Algebraic approximation of mappings into spheres. Mich. Math. J. 34, 119-125 (1987)

8. Bochnak, J., Kucharz, W.: Realization of homotopy classes by algebraic mappings. J. Reine Angew. Math. 377, 159-169 (1987)

9. Bochnak, J., Kucharz, W.: On real algebraic morphisms into even-dimensional spheres. Ann. Math. 128, 415-433 (1988)

10. Bochnak, J., Kucharz, W.: Algebraic models of smooth manifolds. Invent. Math. 97, 585-611 (1989)

11. Bochnak, J., Kucharz, W.: K-Theory of real algebraic surfaces and threefolds. Math. Proc. Camb. Philos. Soc. 106, 471-480 (1989)

12. Bochnak, J., Kucharz, W.: On vector bundles and real algebraic morphisms. In: Real Analytic and Algebraic Geometry, Lecture Notes in Math., vol. 1420, pp. 65-71. Springer, Berlin (1990)

13. Bochnak, J., Kucharz, W.: Polynomial mappings from products of algebraic sets into spheres. J. Reine Angew. Math. 417, 135-139 (1991)

14. Bochnak, J., Kucharz, W.: Vector bundles on a product of real cubic curves. K-Theory 6, 487-497 (1992)

15. Bochnak, J., Kucharz, W.: Algebraic cycles and approximation theorems in real algebraic geometry. Trans. Am. Math. Soc. 337, 463-472 (1993)

16. Bochnak, J., Kucharz, W.: The homotopy groups of some spaces of real algebraic morphisms. Bull. Lond. Math. Soc. 25, 385-392 (1993)

17. Bochnak, J., Kucharz, W.: Elliptic curves and real algebraic morphisms. J. Algebraic Geom. 2, 635-666 (1993)

18. Bochnak, J., Kucharz, W.: The Weierstrass approximation theorem and a characterization of the unit circle. Proc. Am. Math. Soc. 127, 1571-1574 (1999)

19. Bochnak, J., Kucharz, W.: Smooth maps and real algebraic morphisms. Can. Math. Bull. 42, 445-451 (1999)

20. Bochnak, J., Kucharz, W.: The Weierstrass approximation theorem for maps between real algebraic varieties. Math. Ann. 314, 601-612 (1999)

21. Bochnak, J., Kucharz, W.: Line bundles, regular mappings and the underlying real algebraic structure of complex algebraic varieties. Math. Ann. 316, 793-817 (2000)

22. Bochnak, J., Kucharz, W.: Vector bundles on a product of real algebraic curves. Proc. Am. Math. Soc. 133, 1617-1620 (2005)

23. Bochnak, J., Kucharz, W.: Real algebraic morphisms represent few homotopy classes. Math. Ann. 337, 909-921 (2007)

24. Bochnak, J., Kucharz, W.: Algebraic approximation of smooth maps. Univ. Iagell. Acta Math. 48, 9-40 (2010) 
25. Bochnak, J., Kucharz, W., Silhol, R.: Morphisms, line bundles and moduli spaces in real algebraic geometry. Publ. Math. Inst. Hautes Études Sci. 86, 5-65 (1997) [Erratum in Publ. Math. Inst. Hautes Études Sci. 92, 195 (2000)]

26. Bredon, G.E.: Topology and Geometry. Springer, Berlin (1993)

27. Evans, E.G.: Projective modules as fiber bundles. Proc. Am. Math. Soc. 27, 623-626 (1971)

28. Fernando, J.F., Gamboa, J.-M., Ruiz, J.M.: Finiteness problems on Nash manifolds and Nash sets. J. Eur. Math. Soc. 16, 537-570 (2014)

29. Fichou, G., Huisman, J., Mangolte, F., Monnier, J-Ph: Fonctions régulues. J. Reine Angew. Math. 718, 103-151 (2016)

30. Fichou, G., Monnier, J-Ph, Quarez, R.: Continuous functions in the plane regular after one blowing up. Math. Z. 285, 287-323 (2017)

31. Forster, O.: Lectures on Riemann Surfaces. Springer, Berlin (1981)

32. Fossum, R.: Vector bundles over spheres are algebraic. Invent. Math. 8, 222-225 (1969)

33. Ghiloni, R.: On the space of morphisms into generic real algebraic varieties. Ann. Sc. Norm. Super. Pisa Cl. Sci. (5) 5, 419-438 (2006)

34. Ghiloni, R.: Rigidity and moduli space in real algebraic geometry. Math. Ann. 335, 751-766 (2006)

35. Ghiloni, R.: Second order homological obstructions on real algebraic manifolds. Topol. Appl. 154, 3090-3094 (2007)

36. Golubitsky, M., Guillemin, V.: Stable Mappings and Their Singularities. Springer, New York (1973)

37. Huisman, J.: A real algebraic bundle is strongly algebraic whenever its total space is affine. Contemp. Math. 182, 117-119 (1995)

38. Huisman, J.: Correction to "A real algebraic bundle is strongly algebraic whenever its total space is affine". Contemp. Math. 253, 179 (2000)

39. Husemoller, D.: Fibre Bundles, 3rd edn. Springer, New York (1974)

40. Ivanov, N.V.: Approximation of smooth manifolds by real algebraic sets. Russ. Math. Surv. 37(1), 1-59 (1982)

41. Joglar-Prieto, N.: Rational surfaces and regular maps into the 2-dimensional sphere. Math. Z. 234, 399-405 (2000)

42. Joglar-Prieto, N., Kollár, J.: Real abelian varieties with many line bundles. Bull. Lond. Math. Soc. 35, 79-84 (2003)

43. Joglar-Prieto, N., Mangolte, F.: Real algebraic morphisms and del Pezzo surfaces of degree 2. J. Algebraic Geom. 13, 269-285 (2004)

44. Kollár, J., Kucharz, W., Kurdyka, K.: Curve-rational functions. Math. Ann. (2017). https://doi.org/10. 1007/s00208-016-1513-Z

45. Kollár, J., Nowak, K.: Continuous rational functions on real and p-adic varieties. Math. Z. 279, 85-97 (2015)

46. Kosinski, A.A.: Differential Manifolds. Dover, New York (1993)

47. Kucharz, W.: Vector bundles over real algebraic surfaces and threefolds. Compos. Math. 60, 209-225 (1986)

48. Kucharz, W.: Algebraic cycles and vector bundles on real affine threefolds. Manuscr. Math. 60, 211-216 (1988)

49. Kucharz, W.: How to make vector bundles algebraic. C.R. Rep. Sci. Can. 11, 231-235 (1989)

50. Kucharz, W.: Rational maps in real algebraic geometry. Adv. Geom. 9, 517-539 (2009)

51. Kucharz, W.: Complex cycles on algebraic models of smooth manifolds. Math. Ann. 346, 829-856 (2010)

52. Kucharz, W.: Regular versus continuous rational maps. Topol. Appl. 160, 1375-1378 (2013)

53. Kucharz, W.: Regular maps into real Fermat varieties. Bull. Lond. Math. Soc. 451, 1086-1090 (2013)

54. Kucharz, W.: Continuous rational maps into the unit 2-sphere. Arch. Math. (Basel) 102, 257-261 (2014)

55. Kucharz, W.: Approximation by continuous rational maps into spheres. J. Eur. Math. Soc. 16, 15551569 (2014)

56. Kucharz, W.: Complex cycles as obstructions on real algebraic varieties. Glasg. Math. J. 57, 343-347 (2015)

57. Kucharz, W.: Some conjectures on stratified-algebraic vector bundles. J. of Singul. 12, 92-104 (2015)

58. Kucharz, W.: Continuous rational maps into spheres. Math. Z. 283, 1201-1215 (2016)

59. Kucharz, W.: Stratified-algebraic vector bundles of small rank. Arch. Math. (Basel) 107, 239-249 (2016) 
60. Kucharz, W., Kurdyka, K.: Some conjectures on continuous rational maps into spheres. Topol. Appl. 208, 17-29 (2016)

61. Kucharz, W., Kurdyka, K.: Stratified-algebraic vector bundles. J. Reine Angew. Math. (2016). https:// doi.org/10.1515/crelle-2015-0105

62. Kucharz, W., Kurdyka, K.: Linear equations on real algebraic surfaces. Manuscr. Math. (2017). https:// doi.org/10.1007/s00229-017-0925-8

63. Kucharz, W., Kurdyka, K.: Comparison of stratified-algebraic and topological K-theory. arXiv:1511.04238 [math.AG]

64. Kucharz, W., Maciejewski, Ł.: Complexification and homotopy. Homol. Homotopy Appl. 16, 159-165 (2014)

65. Kucharz, W., Rusek, K.: An application of ample vector bundles in real algebraic geometry. Proc. Am. Math. Soc. 139, 1155-1161 (2011)

66. Kucharz, W., Zieliński, M.: Reguluos vector bundles, arXiv:1703.05566 [math.AG]

67. Lee, J.M.: Introduction to Smooth Manifolds. Springer, Berlin (2003)

68. Loday, J.-L.: Applications algébriqes du tore dans la sphére et de $\mathbb{S}^{p} \times \mathbb{S}^{q}$ dans $\mathbb{S}^{p+q}$. In: Algebraic K-Theory II, Lecture Notes in Math., vol. 342, pp. 79-91. Springer, Berlin (1973)

69. Lønsted, K.: An algebraization of vector bundles on compact manifolds. J. Pure Appl. Algebra 2 , 193-207 (1972)

70. Lønsted, K.: Vector bundles over finite CW complexes are algebraic. Proc. Am. Math. Soc. 38, 27-31 (1973)

71. Mangolte, F.: Real algebraic morphisms on 2-dimensional conic bundles. Adv. Geom. 6, 199-213 (2006)

72. Marinari, M.G., Raimondo, M.: Fibrati vettoriali su varietà algebriche definite su corpi non algebricamente chiusi. Boll. Un. Mat. Ital. A 16(5), 128-136 (1979)

73. Milnor, J.W.: Topology from the Differentiable Viewpoint. The University Press of Virginia, Charlottesville (1965)

74. Monnier, J.-P.: Semi-algebraic geometry with rational continuous functions. arXiv:1603.04193 [math.AG]

75. Nowak, K.J.: Some results of algebraic geometry over Henselian rank one valued fields. Sel. Math. New Ser. 28, 455-495 (2017)

76. Ozan, Y.: On entire rational maps in real algebraic geometry. Mich. Math. J. 42, 141-145 (1995)

77. Ozan, Y.: On algebraic K-theory of real algebraic varieties with circle action. J. Pure Appl. Algebra 170, 287-293 (2002)

78. Peng, J., Tang, Z.: Algebraic maps from spheres to spheres. Sci. China Ser. A 42, 1147-1154 (1999)

79. Swan, R.G.: Topological examples of projective modules. Trans. Am. Math. Soc. 230, 201-234 (1977)

80. Swan, R.G.: Vector bundles, projective modules and the K-theory of spheres. In: Algebraic Topology and Algebraic K-Theory, Ann. of Math. Stud., vol. 113, pp. 432-522. Princeton University Press, Princeton (1987)

81. Tognoli, A.: Algebraic Geometry and Nash Functions, Institutiones Math, vol. 3. Academic, New York (1978)

82. Turiel, F.-J.: Polynomial maps and even-dimensional spheres. Proc. Am. Math. Soc. 135, 2665-2667 (2007)

83. Wood, R.: Polynomial maps from spheres to spheres. Invent. Math. 5, 163-168 (1968)

84. Zieliński, M.: Homotopy properties of some real algebraic maps. Homol. Homotopy Appl. 18, 287-294 (2016) 